\title{
Supersymmetric protection and the Swampland
}

\author{
Eran Palti, ${ }^{a, b}$ Cumrun Vafa ${ }^{c}$ and Timo Weigand ${ }^{d}$ \\ ${ }^{a}$ Max-Planck-Institut für Physik (Werner-Heisenberg-Institut), \\ 80805 München, Germany \\ ${ }^{b}$ Department of Physics, Ben-Gurion University of the Negev, \\ Beer-Sheva 84105, Israel \\ ${ }^{c}$ Jefferson Physical Laboratory, Harvard University, \\ Cambridge, MA 02138, U.S.A. \\ ${ }^{d}$ PRISMA Cluster of Excellence and Mainz Institute for Theoretical Physics, \\ Johannes Gutenberg-Universität, \\ 55099 Mainz, Germany \\ E-mail: palti@mppmu.mpg.de, vafa@g.harvard.edu, timo.weigand@cern.ch
}

ABSTRACT: For certain terms in the action, supersymmetry can forbid an infinite number of possible contributions. We study whether such protection can occur in quantum gravity even without sufficient supersymmetry. We focus on whether the superpotential can vanish exactly in four-dimensional $\mathcal{N}=1$ theories, and if the prepotential can be exactly cubic in $\mathcal{N}=2$ theories. We investigate these questions in string theory and find that for almost all known string constructions the corrections allowed by supersymmetry do occur. However, we do find some special settings where all the corrections can be proven to vanish. These examples all share the common feature that they are related, through a certain orbifolding by a discrete gauged R-symmetry element, to a higher supersymmetric theory. Motivated by these results, we propose a Swampland criterion that any theory which enjoys such protection beyond its realised supersymmetry must have a direct connection to a higher supersymmetric theory.

KEYwords: F-Theory, Nonperturbative Effects, Superstring Vacua, Supersymmetric Effective Theories

ARXIV EPRINT: 2003.10452 


\section{Contents}

1 Introduction $\quad 2$

2 String compactifications with generic corrections $\quad 5$

2.1 F-theory vacua with $\mathcal{N}=1$ supersymmetry 5

2.1.1 Instantons on generic Calabi-Yau fourfolds 7

2.1.2 Deformation zero mode lifting by instanton flux 8

2.1.3 Deformation zero mode lifting by D3 brane interactions 9

2.1.4 $\chi\left(Y_{4}\right)=0$ manifolds $\quad 12$

2.1.5 The $E_{8}$ superpotential 13

$\begin{array}{ll}2.2 \text { Other } \mathcal{N}=1 \text { string vacua } & 13\end{array}$

$\begin{array}{ll}2.3 \mathcal{N}=2 \text { prepotentials and their corrections } & 14\end{array}$

3 String compactifications with extra protection $\quad \mathbf{1 5}$

$\begin{array}{ll}3.1 \mathcal{N}=1 \text { theories } & 15\end{array}$

$\begin{array}{lll}3.1 .1 & \text { F-theory vacua with torsional discriminant } & 16\end{array}$

$\begin{array}{lll}3.1 .2 & \text { IIB orbifold vacua with } \mathbb{Z}_{2} \text { fixed points } & 18\end{array}$

$\begin{array}{ll}3.2 \mathcal{N}=2 \text { theories } & 23\end{array}$

3.2.1 Type II string theory on orbifolds 23

3.2.2 IIA string theory on the Enriques Calabi-Yau 24

4 Characteristics of the examples with extra protection 25

$\begin{array}{llr}5 & \text { Discussion } & 26\end{array}$

A Flux lifting of D3-instanton zero-modes in F-theory 28

$\begin{array}{ll}\text { A.1 General mechanism } & 28\end{array}$

A.2 No flux lifting for $B_{3}=\mathbb{P}^{1} \times \mathbb{P}^{2} \quad 28$

$\begin{array}{ll}\text { B Stringy heterotic instantons } & 31\end{array}$

C Superpotentials in heterotic compactifications $\quad 32$

C.1 Standard embeddings 34

$\begin{array}{lll}\text { C.2 Non-standard embeddings } & 36\end{array}$

$\begin{array}{ll}\text { D Type IIA, Type I and M-theory vacua } & 37\end{array}$ 


\section{Introduction}

Supersymmetry is unique as a symmetry which relates external Poincaré charges such as mass to charges with respect to internal symmetries. Such relations are often critical for the existence of certain inequalities between observables of the theory which are precisely saturated for distinguished states. For example, BPS objects exhibit an exact equality between their mass and charge. Closely related to precisely saturated inequalities are precisely vanishing quantities. To name one, a theory which preserves $\mathcal{N}=2$ supersymmetry in four dimensions has an exactly vanishing potential and can admit an exact moduli space. In supersymmetric theories there also exist quantities which enjoy partial protection. For example, the superpotential $W$ in four-dimensional theories with $\mathcal{N}=1$ supersymmetry is allowed to be non-zero but has to be a holomorphic quantity in terms of chiral fields. But can it be that $W=0$ and that quantum corrections do not contribute to $W$ ? Similarly for $\mathcal{N}=2$ theories gauge couplings are determined in terms of prepotentials which are generally not cubic (in flat coordinates) but receive corrections. Could there be gravity theories with $\mathcal{N}=2$ supersymmetry for which all such corrections vanish? In the absence of gravity it is relatively straightforward to construct examples for which such corrections vanish. But quantum gravity is typically far more restrictive as is well known in the context of the Swampland program [1] (for reviews see e.g. [2, 3]). Whether or not the partially protected supersymmetric quantities can have additional vanishing properties in the quantum gravity context is therefore an excellent question for the Swampland program and consequently forms the topic of this paper.

We are particularly interested in whether there exist theories in quantum gravity without the sufficient amount of supersymmetry to guarantee the absence of certain nonperturbative corrections but which nonetheless receive no such corrections. We will see in examples that all quantities which can be corrected by quantum effects are corrected unless the theory is related, in a subtle way, to one with higher supersymmetry responsible for their protection. Let us introduce the quantities we will consider, restricting ourselves to four-dimensional theories throughout this paper.

$\mathcal{N}=1$ supersymmetry. In $\mathcal{N}=1$ supergravities the superpotential $W$ is well-known to receive no perturbative quantum corrections due to holomorphicity in the couplings combined with shift symmetries, and the gauge kinetic function $f$ receives only 1-loop perturbative corrections. However, they both are subject to potentially an infinite number of non-perturbative corrections. In particular, if we suppose that the classical superpotential $W_{\text {cl. }}(\Phi)=0$, the only allowed contributions to the full superpotential take the form

$$
W=\sum_{n, i} A_{n}(\Phi) e^{-a_{n}^{i} \Phi_{i}}
$$

Here $n$ labels the different instanton contributions, while $i$ runs over the chiral superfields. ${ }^{1}$ The $A_{n}$ are (potentially vanishing) holomorphic functions of the fields while the $a_{n}^{i}$ are

\footnotetext{
${ }^{1}$ The exponential terms may also be generated by gaugino condensation in the infrared, but we will focus on the instanton contributions.
} 
constants. Similarly, the gauge kinetic function has an analogous holomorphic expansion, but with an additional potential 1-loop contribution. The question of interest is whether it is possible to have

$$
A_{n}=0 \text { for all } n .
$$

Note that in principle one can split the condition (1.2) into two cases, according to whether it is satisfied for all values of the fields $\Phi_{i}$, or whether there is only a sub-locus in the space of all the massless fields where $W=0$. In $\mathcal{N}=2$ vacua (1.2) is automatically satisfied all over field space.

$\mathcal{N}=\mathbf{2}$ supersymmetry. In $\mathcal{N}=2$ theories, for instance for Type IIA string theory compactified on a Calabi-Yau three-fold, the prepotential $\mathcal{F}$ of the vector multiplet sector contains a term cubic in the superfields, lower polynomial terms and exponential terms,

$$
\mathcal{F}=\mathcal{F}_{\text {polynomial }}(\Phi)+\sum_{n, i} B_{n}(\Phi) e^{-a_{n}^{i} \Phi_{i}},
$$

where the exponential terms are generated by worldsheet instanton effects. We may ask if the infinite series of exponential terms are always present or whether it is possible to have

$$
B_{n}=0 \text { for all } n \text {. }
$$

In $\mathcal{N}=4$ vacua, such as for Type II compactifications on $K 3 \times \mathbb{T}^{2},(1.4)$ is automatically satisfied.

We will refer to the quantities like those appearing on the lefthand side of (1.2) and (1.4) as supersymmetric protected quantities (SPQs) in the following sense: they are of restricted form, but expected to be non-vanishing for generic theories with $\mathcal{N}=1$ or $\mathcal{N}=2$ supersymmetry, respectively, while they vanish in presence of higher supersymmetry. Forbidding an infinite number of operators, without any symmetry responsible for it, would require an infinite amount of accidental cancellations. If there were an infinite number of consistent quantum gravity theories, such accidental cancellations might indeed occur in concrete theories. On the other hand, if the number of consistent theories of quantum gravity is finite, as is widely believed, such a cancellation is extremely unlikely. Invoking a principle of genericity, it is therefore natural to expect that whenever a protected quantity is allowed by supersymmetry, it is non-zero unless it is protected (in some way) by a symmetry.

The goal of this paper is to sharpen this natural expectation by constructing explicit examples of gravitational theories in which the supersymmetric protected quantities vanish. Our findings motivate us to propose a

Supersymmetric Genericity Conjecture. A theory of quantum gravity where a supersymmetric protected quantity (as defined above) vanishes, even though this is not required by the amount of supersymmetry preserved by the theory, must be related to a higher supersymmetric theory.

Note that this conjecture is in line with the general principle that anything which is generically allowed in quantum gravity is 'enforced' and hard to prevent and that there 
occur no accidental vanishings. In the context of quantum gravity theories, for which the finiteness of the allowed possibilities is a Swampland principle, this is plausible: we cannot fine-tune parameters to avoid the generic prediction due to the finiteness of the number of possibilities.

All the examples of quantum gravity theories with vanishing supersymmetric protected quantities as constructed in this paper have the following characteristics: there exists a discrete gauge symmetry $\tilde{\Gamma}$ whose neutral sector is identical to the neutral sector of a higher supersymmetric theory under a discrete R-symmetry $\Gamma{ }^{2}$ Furthermore, if all massless fields in the theory are neutral under $\tilde{\Gamma}$, then the supersymmetric protected quantities vanish everywhere in moduli space.

The examples studied in this paper are in agreement with these characteristics being necessary conditions for the appearance of additional protection beyond the amount of supersymmetry realized in a theory. As we will further exemplify, however, they are not sufficient. Also, while we do not have any explicit realizations, we do not rule out other possible connections to higher supersymmetric theories.

Our guiding principle will be explicit constructions in string theory. Determining whether a supersymmetric protected quantity vanishes is particularly delicate in the case of $\mathcal{N}=1$ supersymmetry. As a first step we will revisit stringy $\mathcal{N}=1$ theories for which the superpotential corrections (1.2) have been proposed to vanish in the literature even though there is no symmetry argument apparent to us which would be responsible for this. We will identify in all such cases subtle effects that have not been accounted for properly and which in fact suggest that the superpotential is non-vanishing. At a technical level, one such effect is the lifting of certain deformation zero-modes for D3-brane instantons in F-theory by quartic fermionic terms in the instanton effective action, as well as the role of stringy spacetime instantons in the heterotic duality frame. Based on this we will construct compactifications free of these subtleties and extract their common properties. We will show that they do satisfy the Supersymmetric Genericity Conjecture, and in fact exhibit the characteristic properties detailed above. Note that this conjecture, if correct, in particular states that exact four-dimensional supersymmetric Minkowski vacua can occur in quantum gravity only in special settings enjoying a protection mechanism which can be traced to some underlying higher supersymmetry.

The examples of theories which we study in this paper fall into two classes, corresponding to whether the supersymmetric protected quantity (SPQ) vanishes for all values of the massless fields - in this case there are no massless fields charged under $\tilde{\Gamma}$ - or only on the sub-locus in field space where the massless fields charged under $\tilde{\Gamma}$ take a zero value. Technically, one way to understand the vanishing of the SPQ is to note that in both cases there exists some duality frame where all the instantons which could create the SPQs exhibit too many fermionic zero-modes. The zero-modes in question are associated with the breaking of a higher supersymmetry by the instanton. They can couple to the massless fields charged under $\tilde{\Gamma}$ and can hence be saturated only if the latter receive a non-zero

\footnotetext{
${ }^{2}$ Note that, unlike $\Gamma$, the discrete gauge symmetry $\tilde{\Gamma}$ is not an R-symmetry. In all examples studied in this work, $\Gamma$ is abelian and $\tilde{\Gamma}$ and $\Gamma$ are isomorphic as groups.
} 
vacuum expectation value. This explains why in absence of such massless charged fields the SPQ vanishes everywhere in moduli space.

The paper is set out as follows. In section 2 we discuss compactifications of string theory which may naively appear to have vanishing SPQs and show that such a conclusion is not justified due to non-perturbative effects that have not been taken into consideration. In section 3 we then study string theory compactifications which are free from the subtleties due to such effects. In section 4 we discuss the common features of these theories and propose a Swampland conjecture based on these features. We discuss our results in section 5. In the appendices we present various technical details as well as further supporting evidence for the picture advocated in this work.

\section{String compactifications with generic corrections}

In this section we present a study of non-perturbative corrections to partially protected quantities in string theory. According to the discussion in the introduction, such corrections are expected to occur unless they are disallowed by a certain type of symmetry. This is in seeming contradiction with candidates reported in the literature for string compactifications without an obvious protection mechanism, but which nonetheless lack, for instance, an $\mathcal{N}=1$ superpotential. We will exemplify that in these cases unaccounted instanton effects can spoil the protection, in agreement with our expectations.

String theory offers a unique window into non-perturbative quantum gravity effects. Specifically, instanton corrections can be understood in terms of extended (non-perturbative) objects in the theory wrapping cycles in the extra dimensions. Some of the brane instantons can be understood as gauge theory instantons [4] (see e.g. [5] for a review), while most instantons have no such gauge theory interpretation and are called stringy instantons. The different types of instantons and their relations through dualities, particularly within the duality orbit involving M-theory and F-theory, are shown in figure 1. Many aspects of stringy instantons relevant to the following analysis, especially the counting of instanton zero-modes, can be found e.g. in the review [6] and references therein.

The work reported in this section involves a significant amount of technical details, as well as new effects which are important for instantons. For example, we prove for the first time the existence of stringy spacetime instantons effects in the heterotic string (which have no gauge theory interpretation) which correct the $\mathcal{N}=1$ superpotential, and propose that certain instanton zero-modes for D-brane instantons in Type II string theory/F-theory can be lifted by quartic interaction terms in presence of suitable spacetime filling branes. Some details and technicalities are presented in the appendices.

\section{$2.1 \quad$ F-theory vacua with $\mathcal{N}=1$ supersymmetry}

F-theory presents possibly the best understood setting for studying non-perturbative effects [7], through its duality with M-theory. Instantons which appear to be at very different footing in other duality frames are all understood as M5-branes wrapping divisors in CalabiYau fourfolds. A generic Calabi-Yau is expected to support many appropriate divisors for 


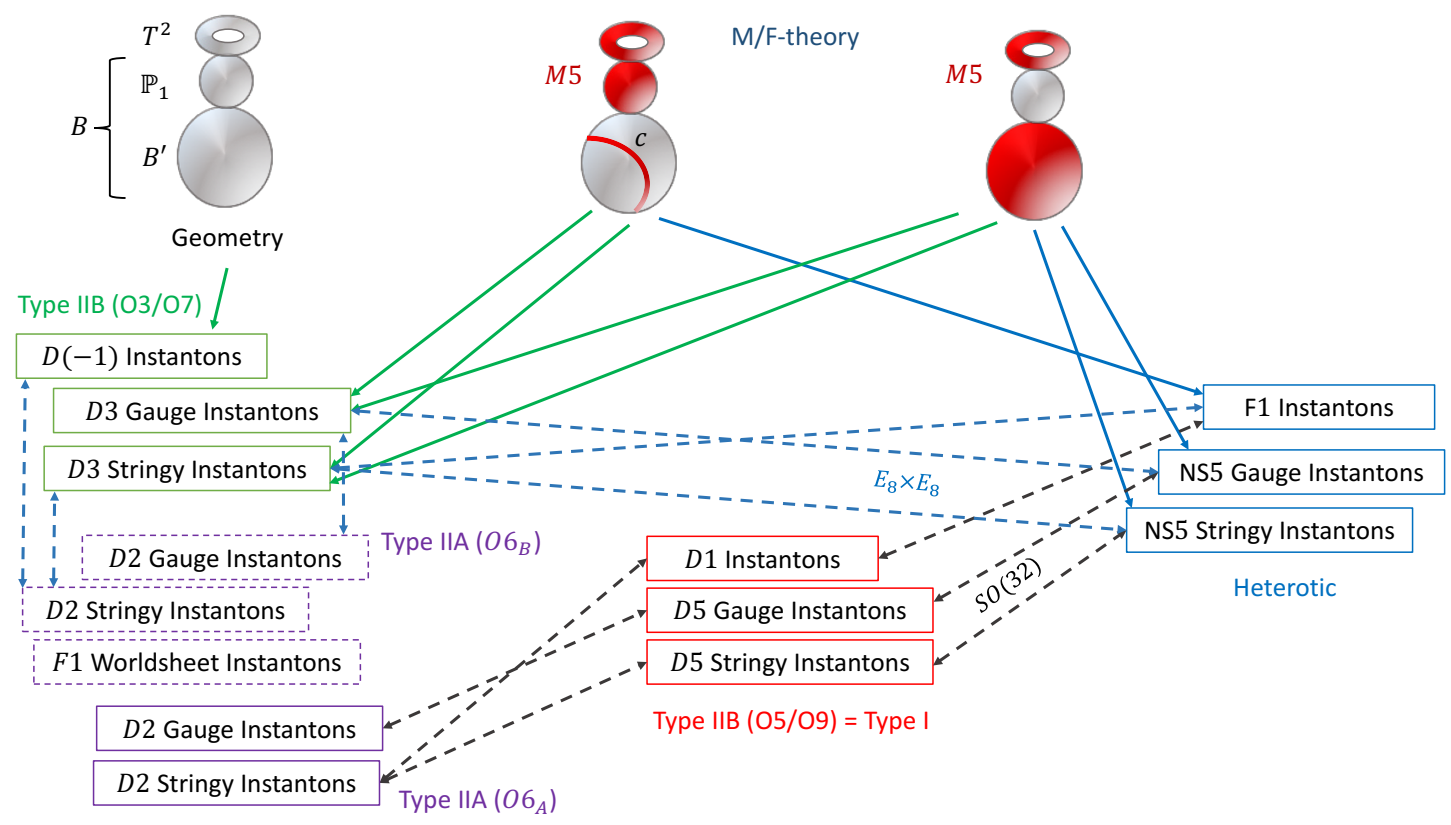

Figure 1. Various types of instantons that appear in string theory, and their relations through dualities given by dashed lines. The dualities can be understood through their M-theory origin, which can also be used to calculate the (neutral) zero-modes of the instantons.

instantons to contribute to the superpotential. In order for this to happen, the instanton must not exhibit any fermionic zero-modes modes besides the two universally present modes $\theta^{\alpha}$ unless they can be saturated in the path integral due to suitable interactions.

The additional zero-modes, if present, fall into two classes: extra Goldstino zero-modes are related to the breaking of a higher supersymmetry by the instanton. Their presence for every instanton in the theory requires a non-generic structure of the theory for which we will construct examples in section 3. All remaining zero-modes admit no such interpretation. Their presence is hence accidental, from the perspective of supersymmetry, and according to the logic proposed in the Introduction, there is no reason why every instanton divisor should exhibit them, in generic situations.

In this section, we will further corroborate this reasoning by re-examining the status of the non-Goldstino zero-modes. The question of whether there are special examples which receive no instanton corrections due to such modes was already preliminarily addressed in the pioneering work [7]. It was proposed that there are certain simple F-theory backgrounds, for example $\mathbb{P}^{3}$ and $\mathbb{P}^{1} \times \mathbb{P}^{2}$, which admit no appropriate divisors for the instantons to contribute to the superpotential. However, further investigations, in particular [8], and new results in this paper show that these examples can, and sometimes certainly do, receive instanton corrections. 


\subsubsection{Instantons on generic Calabi-Yau fourfolds}

Let us first recall the geometric criterion of [7] for instantons to contribute to the superpotential in F-theory. Consider a Calabi-Yau $Y_{4}$ which is elliptically fibered over $B_{3}$,

$$
\pi: Y_{4} \rightarrow B_{3} .
$$

Under F/M-theory duality a D3-brane instanton in F-theory (oftentimes referred to as an E3-brane), along a divisor $D \subset B_{3}$, dualizes to an M5-brane instanton in M-theory wrapping the associated vertical divisor

$$
\hat{D}=\pi^{*}(D)
$$

on $Y_{4}$. In addition to specifying the divisor $D$, one must sum over all admissible instanton flux configurations, which correspond to suitable 3 -form fluxes on $\hat{D}$ [9] or 2-form fluxes on $D[8,10]$.

A sufficient criterion for an instanton along $\hat{D}$ with zero flux to contribute to the superpotential can be stated [7] as

$$
h^{0,0}(\hat{D})=1, \quad h^{i, 0}(\hat{D})=0, \quad i=2,3,4,
$$

where the $h^{i, 0}(\hat{D})$ count the various types of instanton zero-modes as summarized in table 1 . The fermionic zero-modes $\theta^{\alpha}$ (with superpartner $x^{\mu}$ ) are universally present. As will be discussed in section 3.1.1, the modes $\bar{\tau}^{\dot{\alpha}}$ play the role of Goldstino modes for the breaking of a higher supersymmetry felt locally by the instanton, but in generic situations there is no rationale for them to be present for every instanton. Likewise, the remaining zero-modes are not protected by any form of supersymmetry except in special situations such as the ones analyzed in section 3. Generically they are simply associated with internal Wilson line or geometric deformation degrees of freedom of the instanton divisor. ${ }^{3}$

If (2.3) holds, the arithmetic genus

$$
\chi(\hat{D}) \equiv \sum_{i=0}^{4}(-1)^{i} h^{i, 0}(\hat{D})
$$

takes the value

$$
\chi(\hat{D})=1 .
$$

The unit value of $\chi(\hat{D})$ is neither a sufficient nor necessary condition for an instanton to contribute to the superpotential, but it is a crude first order test. One advantage of it is that it is topological and can be easily computed as

$$
\chi(\hat{D})=-\frac{1}{24} \int_{Y_{4}}\left(\hat{D}^{4}+c_{2}\left(Y_{4}\right) \hat{D}^{2}\right)=-\frac{1}{24} \int_{Y_{4}} c_{2}\left(Y_{4}\right) \hat{D}^{2},
$$

\footnotetext{
${ }^{3}$ Apart form these uncharged instanton zero-modes, instantons can carry zero-modes which are charged under the gauge group of the effective theory. For D3-branes instantons these are due to zero-modes in the sector of open strings between the instanton and the spacetime filling branes [11]. For stringy instantons not related to gauge instantons, saturating these zero-modes induces an operator involving charged fields in the instanton contribution [12-15]. For more details we refer e.g. to [6] and references therein.
} 


\begin{tabular}{|c|c|c|c|c|}
\hline zero-modes & statistics & Type IIB & F-theory & M-theory \\
\hline \hline$\left(X_{\mu}, \theta_{\alpha}\right)$ & (bose, fermi) & $h_{+}^{0,0}(\tilde{D})$ & $h^{0}(D, \mathcal{O})$ & $h^{0,0}(\hat{D})$ \\
\hline $\bar{\tau}_{\dot{\alpha}}$ & fermi & $h_{-}^{0,0}(\tilde{D})$ & $h^{0}\left(D,\left.K_{B_{3}}\right|_{D}\right)$ & $h^{1,0}(\hat{D})$ \\
$\gamma_{\alpha}$ & fermi & $h_{+}^{1,0}(\tilde{D})$ & $h^{1}(D, \mathcal{O})$ & \\
\hline$\left(w, \bar{\gamma}_{\dot{\alpha}}\right)$ & (bose, fermi) & $h_{-}^{1,0}(\tilde{D})$ & $h^{1}\left(D,\left.K_{B_{3}}\right|_{D}\right)$ & $h^{2,0}(\hat{D})$ \\
$\chi_{\alpha}$ & fermi & $h_{+}^{2,0}(\tilde{D})$ & $h^{2}(D, \mathcal{O})$ & \\
\hline$\left(c, \bar{\chi}_{\dot{\alpha}}\right)$ & (bose, fermi) & $h_{-}^{2,0}(\tilde{D})$ & $h^{2}\left(D,\left.K_{B_{3}}\right|_{D}\right)$ & $h^{3,0}(\hat{D})$ \\
\hline
\end{tabular}

Table 1. Type IIB and F-theory zero-modes for instantons carrying no instanton flux, as modified from [17]. The M5-brane is wrapping the divisor $\hat{D}$ on an elliptically fibered Calabi-Yau 4 -fold $Y_{4}$ with base $B_{3}$ and canonical bundle $K_{B_{3}}$, while the Type IIB D3 instanton is wrapping the divisor $\tilde{D}$ on the Type IIB Calabi-Yau 3-fold $X_{3}$; $\tilde{D}$ is the double cover of the divisor $D$ given by the projection of $\hat{D}$ to $B_{3}$. The Type IIB counting applies to so-called $O(1)$ instantons, for which $\tilde{D}$ is invariant under the orientifold involution as a divisor, but not pointwise. The last three columns give the homology classes counting the respective types of modes in absence of instanton flux. In the Type IIB case, the homology is split into orientifold even and orientifold odd sectors [17]. The modes counted by $h_{ \pm}^{2,0}(\tilde{D})$ may be lifted by instanton flux [8]. The counting in the fourth column follows from a Leray sequence argument and is valid only if $\hat{D}$ is smooth [18].

where the second equality uses the fact that $\int_{Y_{4}} \hat{D}^{4}=0$ for a vertical divisor. Hence [16]

$$
\chi(\hat{D})=1 \Longleftrightarrow \int_{Y_{4}} c_{2}\left(Y_{4}\right) \hat{D}^{2}=-24 .
$$

This formulation gives a rough first estimate of when we might expect a given Calabi-Yau $Y_{4}$ to contain a divisor appropriate to host an instanton.

To see that generically one expects many such divisors, let the cone of effective divisors on $Y_{4}$ be spanned by the set $\mathcal{C}_{\alpha}$ and write $\hat{D}=\sum_{\alpha} p^{\alpha} \mathcal{C}_{\alpha}$ with $p^{\alpha}$ arbitrary positive integers. The intersection numbers of the $\mathcal{C}_{\alpha}$, as appearing in (2.7), will be some order one integers. Therefore, (2.7) is a single condition on many free integers, which is generically expected to have many possible solutions. ${ }^{4}$ However, there are some exceptions to this admittedly crude expectation, to which we now turn.

\subsubsection{Deformation zero mode lifting by instanton flux}

An example of (an elliptically fibered) Calabi-Yau four-fold $Y_{4}$ which supports no instanton divisors satisfying the condition (2.5) was presented in [7]. It is given by a generic Weierstrass model over the base $B_{3}=\mathbb{P}^{3}$. Such fibration can be realized for instance as a hypersurface in an appropriate $\mathbb{P}^{2}$ bundle over a base $\mathbb{P}^{3}$. M5-brane instantons with a potential contribution to the superpotential in F-theory wrap a divisor $\hat{D}=\pi^{*} D$, where $D=a H$ is a positive integral multiple of the hyperplane class on $\mathbb{P}^{3}$. The cohomology

\footnotetext{
${ }^{4}$ The complication is of course to find solutions over the integers.
} 
groups can be computed explicitly as [7, 19]

$$
h^{i, 0}(\hat{D})=\left(1,0, N_{\mathbb{P}^{3}}(a-4), N_{\mathbb{P}^{3}}(a)\right),
$$

where $N_{\mathbb{P}^{3}}(x)$ denotes the number of polynomials of homogenous degree $x$ in $\mathbb{P}^{3}$. Note that all the additional zero-modes are due to zero-modes other than the Goldstino modes of type $\bar{\tau}$.

It is clear that no divisor $\hat{D}$ with $h^{i, 0}(\hat{D})=(1,0,0,0)$ exists, and in particular $\chi(\hat{D}) \neq$ 1 for any $a>0$. It was therefore proposed in [7] that for such a Calabi-Yau no nonperturbative superpotential is generated.

However, there are two problems with such a conclusion. The first is related to the presence of spacetime-filling D3 branes, necessary to cancel the tadpoles, and will be exemplified in a different setting in section 2.1.3. The second problem is that this conclusion only takes into account instantons which support no instanton flux, and such fluxes can modify the zero mode counting. Indeed, in [8] it was shown that the inclusion of instanton flux leads to the generation of a superpotential, as we will review in appendix A. For example the degree one divisor $a=1$ on $\mathbb{P}^{3}$ corresponds to $D=\mathbb{P}^{2}$ with $h^{2,0}(\hat{D})=0$ and $h^{3,0}(\hat{D})=3$. As discussed in detail in [8] by invoking a weakly coupled orientifold limit, the double cover $\tilde{D}$ of this divisor in the associated Type IIB Calabi-Yau $X_{3}$ allows for instanton flux which lifts all three deformation zero-modes. Hence a non-perturbative superpotential is indeed generated.

\subsubsection{Deformation zero mode lifting by D3 brane interactions}

For the case $B_{3}=\mathbb{P}^{1} \times \mathbb{P}^{2}$, it is still true [7] that there are no divisors which satisfy (2.5). However, this time it is not possible to lift the additional zero-modes with instanton flux using the mechanism of [8]. We show this in appendix A.2.

F-theory on $B_{3}=\mathbb{P}^{1} \times \mathbb{P}^{2}$ (with vanishing background flux) requires the introduction of

$$
N_{D 3}=\frac{1}{24} \chi\left(Y_{4}\right)=822
$$

spacetime-filling D3-branes to cancel the tadpole. We now argue that in the presence of these D3 branes subtle effects can make instantons, even with certain extra zero-modes, contribute to the superpotential. The key point is that there may be certain 4-fermion terms in the instanton action which can saturate the zero mode integral. This effect can saturate the zero-modes counted by $H^{3,0}(\hat{D})$. As we show in appendix A.2, there do exist divisors on $B_{3}=\mathbb{P}^{1} \times \mathbb{P}^{2}$ whose only additional zero-modes are of this type, for instance the instanton with zero mode structure $h^{i, 0}(\hat{D})=(1,0,0,2)$ in equ. (A.4). This suggests the existence of a D3-brane instanton generated superpotential even in this geometry. ${ }^{5}$

\footnotetext{
${ }^{5}$ Note that the effect we are about to describe is different from the well-known interaction of spacetimefilling D3-branes with D3-instanton zero-modes first found in [11]: if the E3-brane already has the correct number of (neutral) zero-modes to contribute to the superpotential, the Pfaffian prefactor of the instanton hits a zero when the instanton intersects the D3-branes. This is due to additional zero-modes between the instanton and the D3 brane. Moving the D3 brane away from the instanton makes these additional strings massive and removes the zero-modes. In our case, the D3-E3 zero-modes participate in quartic couplings involving extra E3-brane deformation modes, which lift both types of zero-modes.
} 
It is informative to study this effect in the Type IIB limit where we can have control over the microscopic physics of the instanton. The map from the F-theory zero-modes to the Type IIB zero-modes is shown in table 1.

Consider the anti-chiral modes $\bar{\chi}^{\dot{\alpha}}$ counted by $h^{3,0}(\hat{D})=h_{-}^{2,0}(\tilde{D})$. These are always paired with a complex bosonic mode $c$ representing a geometric deformation of the instanton cycle $\tilde{D}$ within the Type IIB Calabi-Yau $X_{3}$. In order for the instanton to contribute to the superpotential, it must be possible to saturate the integral

$$
\int d^{4} x d^{2} \theta d^{2} \bar{\chi} d^{2} c e^{-S_{\text {inst }}}
$$

The Grassmann measure $d^{2} \bar{\chi}$ can be saturated through an interaction term in the instanton action of the form $S_{\text {inst }}^{\text {int }}=\bar{\chi} \mathcal{O} \bar{\chi}$, with $\mathcal{O}$ an appropriate operator. Suppose that the D3brane and the instanton are coincident. In such a situation there exist open-string zeromodes at the instanton-brane intersection, which we denote by Grassmann-valued scalars $\lambda_{D E}$ and $\lambda_{E D}$, with subscripts denoting the start and end point of the open string. Then provided there exists a (tree-level) interaction term

$$
S_{\text {inst }}^{\text {int }} \sim \lambda_{D E} \bar{\chi}^{\dot{\alpha}} \bar{\chi}_{\dot{\alpha}} \lambda_{E D}
$$

the zero mode integral $\int d^{4} x d^{2} \theta d^{2} \bar{\chi} d \lambda_{D E} d \lambda_{E D} e^{-S_{\text {inst }}}$ is saturated. Note that this quartic term is accompanied by a cubic coupling

$$
\lambda_{D E} c \lambda_{D E}
$$

involving the bosonic instanton deformation mode $c$.

From the perspective of Type IIB string theory, the interaction term (2.11) corresponds to the zero (external) momentum limit $k \rightarrow 0$ of the disc diagram involving four vertex operator insertions

$$
S_{\text {inst }}^{\text {int }} \sim \lim _{k \rightarrow 0}\left\langle V_{\lambda_{D E}}^{-1 / 2} V_{\bar{\chi}}^{-1 / 2} V_{\bar{\chi}}^{-1 / 2} V_{\lambda_{E D}}^{-1 / 2}\right\rangle_{\text {Disc }} .
$$

The superscripts on the vertex operators give the ghost charge (or picture), which for the disc must sum to -2 . Even without calculating (2.13) explicitly, we note that it is allowed by all the worldsheet selection rules. This follows from the fact that the total U(1) worldsheet charges of the different operators are given by

$$
V_{\bar{\chi}}^{-1 / 2}: Q=\frac{1}{2}, \quad V_{\lambda_{D E} / \lambda_{E D}}^{-1 / 2}: Q=-\frac{1}{2}
$$

and hence cancel in the correlator (2.13). While this does not yet show that the limit of vanishing momentum $k \rightarrow 0$ leads to a contact term in (2.13), at least the correlator is consistent with the worldsheet selection rules.

To go beyond this statement requires performing an explicit CFT computation of the correlator, which is in principle possible for instance in a toroidal setting where $X_{3}=$ $\mathbb{T}_{1}^{2} \times \mathbb{T}_{2}^{2} \times \mathbb{T}_{3}^{2}$ (or an orbifold thereof) and where we can construct the vertex operators explicitly. Associated with each of the three two-tori $\mathbb{T}_{i}^{2}$ there is a $\mathrm{U}(1)_{i}$ selection rule on the worldsheet, the sum of which corresponds to the above total U(1) worldsheet charge 
present on generic Calabi-Yau three-folds. For an E3-brane wrapping, e.g., along $\mathbb{T}_{2}^{2} \times \mathbb{T}_{3}^{2}$, the individual charges are given by (see for example [20])

$$
V_{\bar{\chi}}^{-1 / 2}: Q_{i}=\left(-\frac{1}{2}, \frac{1}{2}, \frac{1}{2}\right), \quad V_{\lambda_{D E} / \lambda_{E D}}^{-1 / 2}: Q_{i}=\left(-\frac{1}{2}, 0,0\right),
$$

leading to a collection of worldsheet charges for the correlator $(2.13)$ of $(-2,1,1)$. Therefore, the operator is forbidden by these individual selection rules. This is to be contrasted with the above smooth $\mathcal{N}=1$ background, where the isometries associated to the $\mathrm{U}(1) \mathrm{s}$ are broken and the total worldsheet $\mathrm{U}(1)$ is the only remaining unbroken such selection rule. To arrive back at such a situation one can consider an orbifold of the torus and insert suitable closed-string twisted mode vertex operators into (2.13) which neutralise the individual U(1) charges. Smoothing the orbifold to a generic Calabi-Yau 3-fold corresponds to giving the twisted modes an expectation value, thereby breaking the $\mathrm{U}(1) \mathrm{s}$ and inducing the operator. We describe such neutralising twisted mode insertions in more detail in section 3.1.2.

We conclude that if there are instantons which intersect the required D3 branes, there can be a four-fermion interaction which is allowed by all selection rules and which would lead to a contribution to the superpotential. At a very heuristic level, this lifting of the zero-modes $\bar{\chi}^{\dot{\alpha}}$ can also be understood by viewing the pair $\lambda_{E D} \lambda_{D E}$ as a bosonic operator $X$ with coupling $X(c+\bar{\chi} \bar{\chi})$. Variation with respect to $X$ fixes the E3-position in the sense that $c=-\bar{\chi} \chi$. Intuitively, requiring that the E3-brane with bosonic deformation mode passes through the spacetime-filling D3 partially rigidifies the E3-brane.

Continuity requires that the lifting effect persists even when the D3-brane brane is moved away from the E3-brane. This results in a position dependent mass $m$ for the zero-modes $\lambda_{E D}$ and $\lambda_{D E}$, modifying the effective interaction to become

$$
X(c+m+\bar{\chi} \bar{\chi}) .
$$

In this picture the only consequence of $m$ is to shift the relation between $\bar{\chi} \bar{\chi}$ and $c$.

Replacing the modes of type $\bar{\chi}^{\dot{\alpha}}$ by the modes $\chi^{\alpha}$ counted by $H_{+}^{2,0}(\tilde{D})$ in the disk diagram (2.13) leads to a correlator whose total worldsheet $\mathrm{U}(1)$ charge is non-vanishing because the $\mathrm{U}(1)$ charge of $V_{\chi}^{-1 / 2}$ is given by $Q=-\frac{1}{2}$. This prevents a lifting of the $\chi^{\alpha}$ modes by the same effect as described for the $\bar{\chi}^{\dot{\alpha}}$ modes. This might have been expected already because unlike the $\bar{\chi}^{\dot{\alpha}}$, the $\chi^{\alpha}$ modes are not accompanied by any bosonic deformation modes. Hence there is no analogue of the intuitive picture that requiring the D3-branes to pass through the E3-divisor partially rigidifies the latter.

We have furthermore no reason to believe that the spacetime-filling D3 branes could lift the zero-modes counted by $h_{ \pm}^{1,0}(\tilde{D})$ on a smooth Calabi-Yau. Indeed, these are Wilson lines and are therefore protected by a discrete gauge symmetry. Note that this conclusion is not in disagreement with the analysis of the toroidal background: consider $\mathbb{T}_{1}^{2} \times \mathbb{T}_{2}^{2} \times \mathbb{T}_{3}^{2}$ and replace the modes $\bar{\chi}$ e.g. by the Wilson line modes $\bar{\gamma}$ associated with the 1-cycles along $\mathbb{T}_{2}^{2}$ (see table 1 ) in the correlator (2.13). This leaves us with $\mathrm{U}(1)$ charges $(0,-1,1)$ for the correlator. This can again be neutralized by insertion of closed string twisted modes on an orbifold. However, for such orbifolds the Wilson line modes are projected out in the first place, in agreement with the fact that the elements of $H^{1,0}(\tilde{D})$ of toroidal orbifolds are 
inherited from the ambient space, while a background preserving $\mathcal{N}=1$ supersymmetry has no 1-cycles.

Note that the interaction term (2.11) is just one of a possible number of four-fermion terms which can lead to instanton contributions to the superpotential. For example, one can have terms involving four fermionic zero-modes of the instanton. However, such a term would require certain conditions on the number of interactions of zero-modes, for example requiring at least two zero-modes.

As alluded to above, we can also interpret the above result from the viewpoint of the Ftheory instanton, where rigid divisors on the base contribute to the superpotential. If there are no rigid divisors, requiring them to pass through points which correspond to some of the positions of the D3 branes in the base of F-theory will rigidify them. This would be our interpretation of the lifting of the corresponding bosonic zero-modes for deformations of the instanton. This is similar to what one sees in topological strings and it would be interesting to study the resulting topological theory which captures such instanton contributions in F-theory. Note also that there were puzzles raised in geometric transitions involving the blow-up of points in F-theory in [21] and one resolution suggested there was the existence of D3 branes contributing to superpotential terms, in accord to what we have argued above.

\subsection{4 $\chi\left(Y_{4}\right)=0$ manifolds}

The results of the previous section suggest that in searching for clean examples of superpotentials with no instanton corrections we should consider F-theory compactifications on manifolds which have no spacetime-filling D3 branes. This corresponds to the geometric condition of a vanishing Euler number,

$$
\chi\left(Y_{4}\right)=0 .
$$

A list of a class of such Calabi-Yau fourfolds can be found in [16]. The examples in this class have $h^{1,1}>20$ which, as discussed in section 2.1.1, suggests, at least in a crude first order approximation, that they should exhibit some divisor which can solve (2.7). An exception to such expectations are Calabi-Yau fourfolds which are a hypersurface in a product of projective spaces, since they were shown to never support an appropriate divisor [7]. However, all such manifolds with $\chi\left(Y_{4}\right)=0$ lead to enhanced supersymmetry and do therefore not represent potential counter-examples to our expectations. To see this, we can even consider the more general class of all complete intersection Calabi-Yau fourfolds, which includes the hypersurfaces in products of projective spaces as a special case. These were fully classified in [22]. It was shown that out of 921,497 such spaces, 15,768 had vanishing Euler number, but they were all product manifolds: $\mathbb{T}^{8}, \mathbb{T}^{2} \times C Y_{3}$ and $\mathbb{T}^{4} \times K 3$. These product manifolds actually then lead to enhanced $\mathcal{N} \geq 2$ supersymmetry. We will consider other examples of Calabi-Yau fourfolds with $\chi\left(Y_{4}\right)=0$ later in the paper.

Note that another way to avoid D3 branes is by saturating the tadpole through closed string background fluxes. However, turning on background fluxes will induce a superpotential [23]. Further, such fluxes can lift instanton zero-modes leading to a non-perturbative 
contribution to the superpotential (see e.g. [6, 24-26] and references therein). It is therefore unclear if this can be a viable route towards a vanishing superpotential vacuum. ${ }^{6}$

\subsubsection{The $E_{8}$ superpotential}

The necessity of spacetime-filling D3 branes can also be important in the context of superpotentials which can have instanton contributions but which may still admit supersymmetric Minkowski vacua. An example of such a superpotential was proposed in [28] (see [29] for the heterotic dual). The superpotential was claimed to be an $E_{8} \theta$-function which was shown to support isolated Minkwoski vacua solutions. The necessary presence of the D3 branes in this example however casts some doubt over whether such solutions exist. This is because including the D3 moduli modifies the superpotential and generally (and possibly inevitably) breaks this $E_{8}$ structure [30]. The fate of the Minkowski vacuum in such a setting remains undetermined, even ignoring fluxed instantons and other effects.

\subsection{Other $\mathcal{N}=1$ string vacua}

Non-perturbative effects in string theory are not fully understood, and even in concrete examples one may not be able to determine the zero-modes of all instanton branes. This means that there remain classes of string compactifications for which we simply cannot definitively answer the question of whether non-perturbative corrections are present or absent. While in some instances certain corrections can be shown to be absent via dualities, proving that all corrections are absent for these theories will not be possible. In this section we will explain why this is the case, and relegate details to the appendix.

The question of whether there exist four-dimensional $\mathcal{N}=1$ supersymmetric compactifications of the heterotic string with no worldsheet instanton corrections to the superpotential was actively studied in the early days of the field [31-33] (see [34-37] for recent work). The question of superpotential corrections was not revisited with as much intensity after the second superstring revolution despite the fact that now we have a better handle also on spacetime non-perturbative effects. Such spacetime instanton corrections can be due to NS5-branes wrapping the whole Calabi-Yau. Only some of these instantons can be understood as gauge theory instantons belonging to the heterotic gauge group. ${ }^{7}$ This already shows that NS5 instantons may indeed contribute to the superpotential. But it is simple to find examples of heterotic string compactifications which have no gauge instantons, and hence at least these classes of NS5-brane instantons can be absent.

Much more difficult to understand are stringy NS5 instantons, which have no gauge theory interpretation. The central problem is the absence of a microscopic theory of NS5 branes which can be used to calculate the zero-modes. This is further complicated by the fact that the NS5 branes can support bundles of the worldvolume two-form, and the instanton sum must include all such possible bundles.

\footnotetext{
${ }^{6}$ If we turn on only D7 worldvolume flux valued in the subspace of $H^{1,1}(\mathrm{D} 7)$ obtained by pullback from the ambient space, then no superpotential is generated but instead a D-term. However, such flux induces D3 charge on the D7 (as otherwise we could not saturate the D3-brane tadpole with its help), and therefore is naturally expected to have an analogous effect as a D3 brane (see, e.g., [27] in a slightly different context).

${ }^{7} \mathrm{~A}$ particularly good handle on this comes from duality with Type I string theory where the instantons are mapped to D5 instantons inside the D9 branes.
} 
To our knowledge, there is no existence proof for stringy NS5 instanton corrections to the superpotential in the heterotic string literature. This leaves open the possibility that there is some obstruction for such corrections to occur. However, in appendix B we present an example of a stringy NS5-brane instanton correction via duality with Ftheory. On the F-theory side we consider a Calabi-Yau fourfold constructed as a smooth Weierstrass model over a threefold base given by a $\mathbb{P}^{1}$ bundle over $\mathbb{P}^{2}$. Because the fibration is smooth, the heterotic dual has no non-Abelian gauge groups and therefore has no gauge theory instantons. As shown in figure 1, M5 branes wrapping the base $\mathbb{P}^{2}$ map to NS5 instantons in the heterotic string [7]. In appendix B we show that for an appropriate twist of the $\mathbb{P}^{1}$ bundle, such branes have the correct number of zero-modes to contribute to the superpotential. This serves as a proof of principle that intrinsically stringy NS5 instantons can contribute to the heterotic superpotential, ruling out any potential general obstruction to such contributions.

The relevance of NS5-brane instantons implies that it is not possible, with current tools, to definitively determine if a given heterotic compactification is free from instanton corrections to the superpotential, at least not directly from the heterotic side. It may be possible to study dual backgrounds in better understood settings, such as F-theory, which brings us back to the discussion of such backgrounds in section 2.1. We will therefore not consider heterotic vacua as possible examples free from corrections.

This prompts the question whether stringy NS5 instantons are the only effect which precludes an example with no corrections to the superpotential. I.e. if we ignore NS5-brane instantons, are there examples with a vanishing superpotential? In appendix $\mathrm{C}$ we study this question, and show that it is very likely that such examples exist. The only ambiguity we could not resolve was due to a new result in [36] showing that there exist loopholes in the Beasley-Witten no-go result against worldsheet instanton corrections in certain setups [33]. If such loopholes apply to the particular examples we consider in appendix $\mathrm{C}$, then they would rule them out as examples with no corrections to the superpotential except for the question of NS5-brane instantons. In any event, we expect that other examples can be found with no corrections except for stringy NS5-brane effects, and believe that the latter are indeed the only completely general effect which can enforce the presence of a superpotential in generic backgrounds.

In appendix D we give a brief account of instanton effects in Type I, Type IIA and M-theory compactifications with four-dimensional $\mathcal{N}=1$ supersymmetry and argue that none of these settings offer sufficient control to definitively prove the absence of instanton corrections to the superpotential. We also comment on flux compactifications.

\section{$2.3 \mathcal{N}=2$ prepotentials and their corrections}

We now briefly consider the situation in $\mathcal{N}=2$ theories. As already discussed in the introduction, we will focus on prepotential terms for $\mathcal{N}=2$ theories. In Type IIA theories they have the structure

$$
\mathcal{F}=\mathcal{F}_{\text {polynomial }}(\Phi)+\sum_{n, i} B_{n}(\Phi) e^{-a_{n}^{i} \Phi_{i}}
$$


Generically there is no reason for the $B_{n}$ to vanish. We ask if this is guaranteed. As is wellknown, general Calabi-Yau manifolds seem to have holomorphic rational curves, and these lead to non-vanishing $B_{n}$ terms above. Indeed for a class of manifolds the non-vanishing of $B_{n}$ is guaranteed on general grounds, in the context of Type IIA compactifications [38]. While this seems to be the generic feature, the exceptions which arise in the conditions discussed in [38] do allow possible cases where the $B_{n}$ may vanish. Manifolds with vanishing Euler characteristic, $\chi=0$, are part of such an exception. Indeed we will construct such examples in the next section where we discuss their relation to higher supersymmetric theories.

\section{String compactifications with extra protection}

The conclusions of section 2 corroborate our expectation that only in very special $\mathcal{N}=1$ supersymmetric string compactifications the superpotential can be guaranteed to vanish exactly, and similarly for corrections to the prepotential in $\mathcal{N}=2$ supersymmetric theories. In the context of $\mathcal{N}=1$ theories, we have discussed a number of natural, and previously proposed, candidates for such theories and showed that in all cases some unaccounted effects cast doubt on the conclusion that instanton corrections are really absent. One may consider the possibility that theories where the infinite number of potential instanton corrections are absent are in the Swampland. However, this is not the case, and one aim of this section is to prove this by construction. We will discuss various examples where the $\mathcal{N}=1$ superpotential is protected against non-perturbative corrections in a way expected for theories with $\mathcal{N} \geq 2$ supersymmetry. Similarly we will provide examples of $\mathcal{N}=2$ theories whose prepotentials receive no corrections in a way one would usually expect for theories with $\mathcal{N} \geq 4$ supersymmetry.

The basic structure of all the examples we will consider is as follows: we start with a higher supersymmetric theory and consider orbifolding it by a discrete R-symmetry group $\Gamma$, which breaks the supersymmetry to the desired one $(\mathcal{N}=1$ or $\mathcal{N}=2)$. We find examples where $\Gamma$ has no fixed points and where over all of moduli space the corrections are absent to all orders; we are able to relate this vanishing to the vanishing properties of the higher supersymmetric theory. We also find examples of this type where $\Gamma$ does have fixed points, leading to some additional massless fields. We show that as long as we do not give a VEV to these massless fields the vanishing condition persists. But if these fields do acquire a VEV, the protection against the corrections is no longer guaranteed.

Below we give a number of examples of these types of construction for both $\mathcal{N}=1$ as well as $\mathcal{N}=2$ theories. Even though some of the constructions and the arguments for the observed vanishing behaviour are technical (especially in the $\mathcal{N}=1$ case), they all share the above mentioned simple structure.

\section{1 $\mathcal{N}=1$ theories}

We begin with theories preserving four-dimensional $\mathcal{N}=1$ supersymmetry, but which still receive no corrections to the superpotential. There are two types of such constructions, corresponding to whether the superpotential vanishes everywhere in field space or only on 


\begin{tabular}{|c|c|}
\hline $\mathcal{N}=1$ & $\mathcal{N}=1^{\prime}$ \\
\hline$\theta^{\alpha}$ & $\tau^{\alpha}$ \\
\hline $\bar{\theta}^{\dot{\alpha}}$ & $\bar{\tau}^{\dot{\alpha}}$ \\
\hline
\end{tabular}

Table 2. Goldstino modes of an instanton in Type II orientifolds.

a sub-locus of the space of massless fields where it admits a supersymmetric Minkowski vacuum.

\subsubsection{F-theory vacua with torsional discriminant}

Our first example of a class of $\mathcal{N}=1$ theories with an exactly vanishing superpotential is due to the presence of the Goldstino instanton zero-modes $\bar{\tau}^{\dot{\alpha}}$ in table 1 . They are related to supersymmetry as follows:

Suppose first that we can take a weakly coupled Type IIB orientifold limit. As explained in [6] and references therein, the background locally preserves an $\mathcal{N}=2$ supersymmetry algebra, which can be decomposed schematically as $\mathcal{N}=2 \supset(\mathcal{N}=1) \oplus\left(\mathcal{N}=1^{\prime}\right)$. The system of spacetime filling D-branes and O-planes preserves the first $\mathcal{N}=1$ subgroup (with supercharges $Q^{\alpha}$ and $\bar{Q}^{\dot{\alpha}}$ ) and breaks the $\mathcal{N}=1^{\prime}$ superalgebra (with supercharges $Q^{\prime \alpha}$ and $\left.\bar{Q}^{\prime \dot{\alpha}}\right)$. Due to its pointlike localisation within $\mathbb{R}^{1,3}$, a half-BPS instanton preserves a different $\mathcal{N}=1$ subalgebra generated by $Q^{\prime \alpha}$ and $\bar{Q}^{\dot{\alpha}}$. The Goldstino modes associated with the spontaneous breaking of the orthogonal complement by the instanton are the modes $\theta^{\alpha}$ and $\bar{\tau}^{\dot{\alpha}}$ as displayed in table 2 .

The modes $\bar{\tau}$ can therefore only be absent if the instanton locally feels the explicit breaking of $\mathcal{N}=2$ to $\mathcal{N}=1$ by the background. This requires, as a necessary condition, that it intersects the system of branes and O-planes suitably. ${ }^{8}$

There is a more general way to understand when the instanton zero-modes $\bar{\tau}$ are absent from the F-theory perspective. In F-theory, the system of D-branes and O-planes maps to the discriminant locus $\Delta=12 \bar{K}_{B_{3}}$ (with $\bar{K}_{B_{3}}=K_{B_{3}}^{-1}$ the anti-canonical bundle of $B_{3}$ ). Requiring an intersection of the instanton with the system of branes in F-theory therefore means that (see the discussion in $[50,51]$ )

$$
\left.K_{B_{3}}\right|_{D} \neq \mathcal{O}_{D}
$$

If this does not hold, i.e. if $\left.K_{B_{3}}\right|_{D}=\mathcal{O}_{D}$, the counting of zero-modes in table 1 yields one pair of zero-modes $\bar{\tau}^{\dot{\alpha}}$ because $\operatorname{dim}\left(H^{0}\left(D,\left.K_{B_{3}}\right|_{D}\right)\right)=1$.

More generally, such modes are guaranteed to be present for every instanton divisor $D$ only if $\left.K_{B_{3}}\right|_{D}$ has a section, which means that it must be a non-negative line bundle and hence the anti-canonical bundle must satisfy $\left.c_{1}\left(\bar{K}_{B_{3}}\right)\right|_{D} \leq 0$, for every divisor class $D$. The case $\left.c_{1}\left(\bar{K}_{B_{3}}\right)\right|_{D}<0$ for every divisor class $D$ can be excluded because for an elliptic fibration

\footnotetext{
${ }^{8}$ There are two possibilities: if the instanton wraps the same cycle as a spacetime-filling brane, the $\bar{\tau}$ modes are lifted by an ADHM-type interaction [39, 40], even if it is not a gauge instanton [41, 42]. The second possibility [43-46] is that the instanton wraps a divisor which, at generic points in its moduli space [47-49], is invariant under the orientifold involution, though not pointwise (a so-called $O(1)$ instanton); this implies that it must intersect the orientifold over a curve.
} 
to exist, the anti-canonical bundle $\bar{K}_{B_{3}}$ must not be negative. ${ }^{9}$ The only possibility leading to Goldstino modes $\bar{\tau}$ for every instanton divisor is that $\bar{K}_{B_{3}}$ and hence also $K_{B_{3}}$ is either trivial or torsional. Since the first case leads to supersymmetry enhancement in an obvious way, we must require the latter. In this case we must require in addition that $\left.K_{B_{3}}\right|_{D}=\mathcal{O}_{D}$ for every divisor $D$ rather than a torsional line bundle on $D$ since such a torsional line bundle has no non-trivial sections.

These two conditions are achieved in F-theory on a freely acting quotient Calabi-Yau four-fold of the form

$$
Y_{4}=\frac{X_{3} \times \mathbb{T}^{2}}{\Gamma}, \quad \Gamma=\mathbb{Z}_{k}
$$

where $X_{3}$ is a Calabi-Yau threefold and $k \in\{2,3,4,6\}$. A simple way to construct such vacua is to let $\Gamma$ act as an involution ${ }^{10}$ on $\mathbb{T}^{2}$ and act freely on $X_{3}$. There are many examples of Calabi-Yau threefolds with such freely acting symmetries. The classification of such symmetries for complete intersection Calabi-Yau manifolds reports 166 cases for $\Gamma=\mathbb{Z}_{2}[52]$. Note that the four-fold $Y_{4}$ is a non-trivial elliptic fibration over $B_{3}=X_{3} / \Gamma$.

In fact, for constructions of type (3.2),

$$
\operatorname{Tor}\left(H^{2}\left(B_{3}, \mathbb{Z}\right)\right)=\tilde{\Gamma} \oplus R, \quad \tilde{\Gamma}=\mathbb{Z}_{k},
$$

where $R$, if non-zero, is inherited from $\operatorname{Tor} H^{2}\left(X_{3}, \mathbb{Z}\right)$, while $\tilde{\Gamma}$ is induced by taking the free quotient. Indeed, taking a freely-acting quotient leads to non-trivial torsional onecycles, and $\operatorname{Tor}\left(H_{1}\left(B_{3}, \mathbb{Z}\right)\right)=\operatorname{Tor}\left(H^{2}\left(B_{3}, \mathbb{Z}\right)\right)$. Importantly, since $c_{1}\left(X_{3}\right)=0$, the only contribution to $c_{1}\left(K_{B_{3}}\right)$ comes from taking the quotient. Therefore

$$
c_{1}\left(K_{B_{3}}\right) \in \tilde{\Gamma} .
$$

It follows that the restriction $\left.K_{B_{3}}\right|_{D}$ to any divisor $D$ on $B_{3}$ must be trivial: otherwise it would define an element in $\operatorname{Tor}\left(H^{4}\left(B_{3}, \mathbb{Z}\right)\right)$, but this latter group is unrelated to taking the quotient by $\Gamma$. Hence, if $\operatorname{Tor}\left(H^{4}\left(B_{3}, \mathbb{Z}\right)\right)$ is non-trivial, it must be inherited from the parent Calabi-Yau $X_{3}$ appearing in (3.2). This implies that $\left.K_{B_{3}}\right|_{D}=\mathcal{O}_{D}$. We have therefore established the presence of the Goldstino zero-modes $\bar{\tau}$ counted by

$$
\operatorname{dim}\left(H^{0}\left(D,\left.K_{B_{3}}\right|_{D}\right)\right)=1
$$

preventing the generation of a superpotential everywhere in field space. ${ }^{11}$

\footnotetext{
${ }^{9}$ Recall that this is because the functions $f$ and $g$ in the Weierstrass model $y^{2}=x^{3}+f x z^{4}+g z^{6}$ describing an elliptic fibration are sections of $\bar{K}_{B_{3}}^{4}$ and $\bar{K}_{B_{3}}^{6}$, and therefore $\bar{K}_{B_{3}}$ must not be a negative line bundle as otherwise no such sections exist. Likewise, $\left.c_{1}\left(\bar{K}_{B_{3}}\right)\right|_{D}<0$ except for some $D$ for which $\left.c_{1}\left(\bar{K}_{B_{3}}\right)\right|_{D}=0$ cannot lead to an $\mathcal{N}=1$ supersymmetric background.

${ }^{10}$ For $\Gamma=\mathbb{Z}_{3}, \mathbb{Z}_{4}, \mathbb{Z}_{6}$ the complex structure of $\mathbb{T}^{2}$ is fixed at a value compatible with taking the quotient.

${ }^{11}$ Instead [47], the instantons generate higher derivative F-terms [53, 54] of the form

$$
\int d^{4} x d^{2} \theta \bar{D} \bar{\Phi} \bar{D} \bar{\Phi}(\ldots) e^{-S(\Psi)} .
$$

Here the $\mathcal{N}=1$ superfields $\Psi$ and $\Phi$ combine into an $\mathcal{N}=2$ hypermultiplet in the parent Type II theory prior to orientifolding [49].
} 
The same conclusion is reached in the orientifold picture (available for $\Gamma=\mathbb{Z}_{2}$ ) by noting that the double cover of the instanton divisor $D$ is the sum of two non-intersecting divisors on $X_{3}$ exchanged by the freely acting involution. Hence one linear combination of the Goldstino modes survives in F-theory.

We conclude that instanton corrections to the superpotential are absent everywhere in field space in $\mathcal{N}=1 \mathrm{~F}$-theory compactifications when the fibration is purely torsional, i.e. $c_{1}\left(K_{B_{3}}\right) \in \operatorname{Tor} H^{2}\left(B_{3}, \mathbb{Z}\right)$. The reason for the vanishing of the superpotential is not just the presence of arbitrary zero-modes, but of the Goldstino modes associated with an underlying higher supersymmetry. This is a first encounter for $\mathcal{N}=1$ theories of the interplay between the absence of non-perturbative effects and the presence of torsional cohomology. Note that the torsional group $\tilde{\Gamma}$ in (3.3) translates into a discrete gauge symmetry in the $\mathcal{N}=1 \mathrm{~F}$ theory compactification $[55,56]$. In section 4 we will identify this gauge symmetry as a key common feature at least of the theories with vanishing supersymmetric protected quantities, in this case the superpotential, found in this paper.

\subsubsection{IIB orbifold vacua with $\mathbb{Z}_{2}$ fixed points}

In this section we give an example of an exactly vanishing superpotential on a certain sub-locus of field space. The absence of the superpotential is due to additional zero-modes for all instantons. As before these can be interpreted as certain Goldstino modes, though this interpretation is less manifest.

The background is chosen as the orientifold of an orbifold $\mathbb{T}^{6} / \Gamma$. The combined action of $\Gamma$ and the orientifold involution $\sigma$ breaks the supersymmetry from $\mathcal{N}=8$ to $\mathcal{N}=1$. This explicit supersymmetry breaking is a global effect, to the extent that locally away from all fixed point loci of the orbifold and orientifold an object still probes the original supersymmetry. More generally, if an instanton wraps a cycle which intersects some of the fixed point loci, it probes only a subset of the original supercharges. This occurs whenever a subset of the orbifold and orientifold elements acts freely on the instanton. In principle, this subset can be different for different instantons. As we will propose, the spontaneous breaking of these supercharges by the instanton leads to the appearance of a certain number of instanton Goldstino zero-modes. These zero-modes are protected except for possible couplings to the massless twisted sector fields of the orbifold. As these acquire a VEV, the zero-modes can be lifted and a superpotential can in principle be generated.

Our notation is as in [57]: we consider an orbifold quotient by a discrete group $\Gamma=$ $\mathbb{Z}_{2} \times \mathbb{Z}_{2}$ acting on the three torus factors of $\mathbb{T}^{6}=\mathbb{T}_{1}^{2} \times \mathbb{T}_{2}^{2} \times \mathbb{T}_{3}^{2}$ with complex coordinates $\left(z_{1}, z_{2}, z_{3}\right)$. The action of a $\mathbb{Z}_{2}$ is specified by $\left(\epsilon_{1} \delta_{1}, \epsilon_{2} \delta_{2}, \epsilon_{3} \delta_{3}\right)$ with $\epsilon_{i} \in\left\{0, \frac{1}{2}\right\}$ and $\delta_{i}= \pm$ as

$$
z_{i} \rightarrow \delta_{i} z_{i}+\epsilon_{i}
$$

The complex coordinates decompose into real components as $d z_{i}=d x_{i}+\tau d x_{i+3}$ (we take $\tau=\sqrt{-1}$ unless otherwise specified).

We consider a Type IIB orientifold of the orbifold modded out by the $\mathbb{Z}_{2}$ symmetries generated by

$$
\theta:(0+, 0-, 0-), \quad \theta^{\prime}:(0-, 0+, 0-) .
$$


Unlike [58], where this orbifold was studied, we do not turn on discrete torsion, which means the Hodge numbers are $h^{1,1}=3$ and $h^{2,1}=51$. These arise from $h^{1,1}=h^{2,1}=3$ universal modes of $\mathbb{T}_{1}^{2} \times \mathbb{T}_{2}^{2} \times \mathbb{T}_{3}^{2}$ and 48 twisted modes. We then further orientifold by a holomorphic involution (see for example $[59,60]$ )

$$
\sigma:(0-, 0-, 0-) \text {. }
$$

This leads to 64 O3-planes located at the fixed points of $\sigma$, as well as 12 O7-planes located at the fixed points of $\sigma \theta, \sigma \theta^{\prime}$ and $\sigma \theta \theta^{\prime}$. We place $8 \mathrm{D} 7$ branes on top of each of the O7 planes to cancel the tadpoles, giving a gauge group $\mathrm{SO}(8)^{12}$. We also need to add $32 \mathrm{D} 3$ branes to cancel the $\mathrm{O} 3$ charges, which can be placed anywhere on the orbifold.

The possible instantons contributing to the superpotential come from D3 branes wrapping holomorphic divisors. Since $h^{1,1}=3$, these instantons are associated purely to the untwisted sector. The generators of the divisor group are the four-cycles on $\mathbb{T}^{6}$ which are not projected out by the quotient by $\Gamma$ and $\sigma$.

To study these, let us introduce the notation

$$
D_{i}=\frac{1}{|\Gamma|} \sum_{g \in \Gamma} g \mathbb{D}_{i}, \quad \mathbb{D}_{i}=p_{i} \times \mathbb{T}_{j}^{2} \times \mathbb{T}_{k}^{2},
$$

where $\mathbb{D}_{i}$ is a divisor on $\mathbb{T}_{1}^{2} \times \mathbb{T}_{2}^{2} \times \mathbb{T}_{3}^{2}$ located at the point $p_{i}$ on $\mathbb{T}_{i}^{2}$ (and $(i, j, k)$ is a permutation of $(1,2,3))$. Then the divisor group of the orientifolded background is generated by

$$
\mathcal{D}_{i}=\frac{1}{2}\left(D_{i}+D_{i}^{\prime}\right), \quad D_{i}^{\prime}=\sigma D_{i} .
$$

Note in particular that the orientifold image of the point $p_{i}$ is the reflected point $p_{i}^{\prime}=\sigma p_{i}$, and $p_{i}^{\prime} \neq p_{i}$ unless $D_{i}$ is located at one of the 4 orientifold fixed planes on $\mathbb{T}_{i}^{2}$.

Before discussing more general cycles than the generators $\mathcal{D}_{i}$, let us note that for generic position of $p_{i}$, a subset of the orbifold and orientifold group elements acts freely on $\mathcal{D}_{i}$. For example, consider the divisor $\mathcal{D}_{1}$, wrapping the second and third torus with $p_{1}$ away from the fixed points on $\mathbb{T}_{1}^{2}$. The freely acting elements are those which act as inversion on $\mathbb{T}_{1}^{2}$ and hence map $p_{1}$ to $-p_{1}$. These are the elements $\theta^{\prime}, \theta \theta^{\prime}, \sigma$ and $\sigma \theta$. Note that these include the generators of the orientifold involution, $\sigma$, and of one of the $\mathbb{Z}_{2}$ orbifold factors, $\theta^{\prime}$, even though the set of freely-acting elements on the instanton does not form a group $\mathbb{Z}_{2} \times \mathbb{Z}_{2}$. By contrast, the non-freely acting group elements are those which act as inversion along $\mathbb{T}_{2}^{2} \times \mathbb{T}_{3}^{2}$ or separately on $\mathbb{T}_{2}^{2}$ and $\mathbb{T}_{3}^{2}$ without affecting $\mathbb{T}_{1}^{2}$. These are the elements $\theta, \sigma \theta^{\prime}$ and $\left(\sigma \theta^{\prime}\right) \theta$.

The fact that some elements of the combined orbifold and orientifold action act freely on the instanton implies that the instanton locally probes a higher supersymmetry than the $\mathcal{N}=1$ supersymmetry. This should result in the appearance of Goldstino instanton zero-modes beyond the universal modes $\theta^{\alpha}$ because the instanton spontaneously breaks this higher supersymmetry. The situation is similar to the appearance of Goldstino modes in the setup of section 3.1.1, with the difference that first we expect more Goldstino modes since the instanton probes a higher local supersymmetry than in this example, and second 
the specific embedding of the higher supersymmetry into the original $\mathcal{N}=8$ differs for the different choices of cycles $\mathcal{D}_{i}, i=1,2,3$.

In fact, the instanton zero-modes for an instanton along $\mathcal{D}_{i}$ at generic position are the modes

$$
\left(x^{\mu}, \theta^{\alpha}, \bar{\tau}^{\dot{\alpha}}\right), \quad\left(c_{i}, \chi_{i}^{\alpha}, \bar{\chi}_{i}^{\dot{\alpha}}\right) .
$$

Apart from the four universal fermionic modes $\theta^{\alpha}$ and $\bar{\tau}^{\dot{\alpha}}$ the instanton exhibits four fermionic deformation modes associated with its location on $\mathbb{T}_{i}^{2}$. Note that the zero-modes associated with the Wilson lines along the wrapped tori prior to taking the quotient are projected out.

A general D3-brane instanton wraps a divisor of the form

$$
\mathcal{D}=\sum_{i=1}^{3} a_{i} \mathcal{D}_{i} .
$$

If each $D_{i}$ and $D_{i}^{\prime}$ in (3.11) are locally identical, $\mathcal{D}$ is invariant pointwise under the orientifold (corresponding to an $\operatorname{Sp}(1)$ instanton) ${ }^{12}$ For more general $\mathcal{D}$, the situation is similar to the individual $\mathcal{D}_{i}$ at generic position discussed above, and the instanton in particular always exhibits the zero-modes $\bar{\tau}^{\dot{\alpha}}$ which must lifted for a superpotential to be generated. To see when this can happen, note that if at least two $a_{i}$ are non-zero, one can deform the formal sum of divisors in $\mathcal{D}$ into single divisor which is invariant as a whole (though not pointwise) under the orientifold involution. For the potential contribution to the superpotential it is immaterial in which phase we consider the instanton (in fact, integrating over the instanton moduli space requires us to sum up both types of contributions). We therefore focus on the generic situation, corresponding to a smooth invariant divisor, which we call $\tilde{\mathcal{D}}$.

The invariant bound state $\tilde{\mathcal{D}}$ locally feels the orientifold action and we are left with only one universal mode system $\left(x^{\mu}, \theta^{\alpha}\right)$ as well as a certain number of orientifold odd components of type $\left(c, \bar{\chi}^{\dot{\alpha}}\right)$. The latter are, in this sense, remnants of Goldstino zeromodes of the constituent cycles $D_{i}$ and $D_{i}^{\prime}$. This implies that we expect them to be protected except for possible couplings involving the massless twisted closed string fields. Once the latter acquire a VEV, the local structure of supersymmetry is broken and the zero-modes can in principle be lifted. ${ }^{13}$ Therefore the interactions responsible for the lifting of the Goldstino modes must involve the twisted massless fields, and the superpotential must have twisted-mode prefactors. Since we must introduce spacetime filling D3 branes to cancel the $\mathrm{O} 3$ charges, the deformation modes could, for instance, be lifted by the mechanism of section 2.1.3. But, again, this would lead to twisted modes as instanton prefactors.

\footnotetext{
${ }^{12}$ The contribution of such an instanton to the superpotential, if non-vanishing, involves the $\mathrm{SO}(8)$ gauge sector fields located at the orientifold planes. Apart from this, the relevant aspects of the following discussion about the lifting of the remaining zero-modes applies to this type of instantons as well and we therefore do not need to consider these separately.

${ }^{13}$ Note that the closed string sector massless modes correspond to complex structure deformations of the orbifold.
} 
The crucial question is how many twisted mode expectation values are required to lift all the zero-modes. Suppose that all instantons could contribute to the superpotential with only two or more twisted mode insertions; under this assumption there would exist a supersymmetric Minkwoski vacuum with an exactly vanishing superpotential. Specifically, all instanton terms in the superpotential would take the form

$$
\left(\prod_{p} \Phi_{p}^{i_{p}}\right) e^{-T}
$$

where $T$ denotes the superfield controlling the volume of the cycle wrapped by the instanton. The $\Phi_{p}$ are twisted modes, which must be inserted $i_{p}$ times, and the worldsheet $\mathrm{U}(1)$ charge selection rules discussed in section 2.1 .3 would imply that, by the above assumption

$$
\sum_{p} i_{p} \geq 2
$$

The superpotential (3.14) admits a solution to $W=d W=0$ on the locus $\Phi_{p}=0$. Note that the existence of this vacuum is independent of the Kähler potential, which means that we need not worry about how to calculate the kinetic terms for the twisted modes. The rest of this section is dedicated to showing that the condition (3.15) indeed holds.

We will only be concerned with the charges of the vertex operators under the three worldsheet $\mathrm{U}(1)$ selection rules associated to the tori. In the $(-1 / 2)$ picture, the vertex operators e.g. for deformation modes along $\mathbb{T}_{1}^{2}$ have the following charges (see e.g. [20]):

$$
V_{\bar{\chi}_{1}}^{(-1 / 2)}:\left(-\frac{1}{2}, \frac{1}{2}, \frac{1}{2}\right) \text {. }
$$

The massless closed-string twisted modes in the $(-1,-1)$ picture have charges (see, for example [61])

$$
V^{(-1,-1)}:\left(2 \theta_{1}, 2 \theta_{2}, 2 \theta_{3}\right),
$$

where the $\theta_{i}$ are the orbifold twists and $\sum_{i} \theta_{i}=1$. The overall factor of 2 appears because for closed-string operators the twist fields appear in both left and right-moving sectors. So in the case of the orbifold (3.8) we have in particular

$$
V_{1}^{(-1,-1)}:(1,1,0), \quad V_{2}^{(-1,-1)}:(1,0,1), \quad V_{3}^{(-1,-1)}:(0,1,1) .
$$

To achieve charge -2 , as required for disc diagrams, we may have to picture change some of the vertex operators. This is done by the prescription [62] (see also for example [61,63])

$$
V^{(c+1)}(w)=\lim _{z \rightarrow w} e^{\phi(z)} T_{F}(z) V^{(c)}(w)
$$

where $\phi$ is the (bosonised) ghost, and the (internal part of) $T_{F}$ is

$$
T_{F}(z)=\sum_{i=1}^{3}\left[\partial \bar{z}_{i} \psi_{i}(z)+\partial z_{i} \bar{\psi}_{i}\right] .
$$


Here the $\psi_{i}$ and $\bar{\psi}_{i}$ are the bosonised worldsheet spinors along the torus directions and have charges +1 and -1 , respectively. At least purely with respect to the $\mathrm{U}(1)$ charges, each picture changing adds \pm 1 to one of the $\mathrm{U}(1)$ charges.

We would like to consider lifting the deformation modes $\bar{\chi}_{1}$ along $\mathbb{T}_{1}^{2}$ through some operator

$$
\mathcal{O} \bar{\chi}_{1} \bar{\chi}_{1}
$$

The piece $\bar{\chi}_{1} \bar{\chi}_{1}$ has $\mathrm{U}(1)$ charges $(-1,1,1)$ and ghost charge -1 . It is therefore not possible for $\mathcal{O}$ to be an insertion of just a single twisted vertex operator in the appropriate $(0,-1)$ picture. Further, since each instanton has just one deformation mode per torus, it is not possible to write a quartic interaction. On the other hand, $\mathcal{O}$ can in principle correspond e.g. to a term quadratic in the massless closed string twisted vertex operators, at least to the extent that such correlators do not violate any worldsheet $\mathrm{U}(1)$ charge.

In addition to such direct couplings to the closed string massless twisted sector, couplings involving the spacetime filling D3 branes could lift the deformation modes $\bar{\chi}_{i}$. Indeed, if we consider the $(0,0)$ picture twisted vertex operators

$$
V_{1}^{(0,0)}:(1,0,-1), \quad V_{2}^{(0,0)}:(1,-1,0),
$$

then the disc correlator

$$
\left\langle V_{1}^{(0,0)} V_{2}^{(0,0)} V_{\lambda_{D E}}^{-1 / 2} V_{\bar{\chi}}^{-1 / 2} V_{\bar{\chi}}^{-1 / 2} V_{\lambda_{E D}}^{-1 / 2}\right\rangle_{D i s c}
$$

is neutral under all the $\mathrm{U}(1)$ charges. This gives an explicit realisation of the effect discussed in section 2.1.3 (but does not ensure the further required condition of a non-trivial zero momentum limit). Again we see that the operator $\mathcal{O}$ in (3.21) involves at least two twisted mode insertions, thereby ensuring (3.15).

We conclude that all instanton contributions to the superpotential are such that $W=$ $d W=0$ on the sub-locus in moduli space where the (closed string) massless twisted sector fields take a vanishing expectation value. The origin of this vanishing result are the zeromodes which are related to the individual deformations of the divisors $\mathcal{D}_{i}$ generating the divisor group of the orbifold. We interpret these zero-modes as Goldstino modes of the instanton spontaneously breaking a certain higher supersymmetry probed locally by the instanton.

It is instructive to contrast the orbifold action (3.8) without discrete torsion to its cousin with discrete torsion [58], giving rise to Hodge numbers $h^{1,1}=51, h^{2,1}=3$. The twisted sector now includes rigid divisors without any deformation moduli. Instantons along such divisors contribute to the superpotential $[64,65]$ even without the need to invoke the mechanisms of zero mode saturation described above. See $[20,66]$ for the mirror dual Type IIA setting, and $[65,67]$ for a systematic study of other Type IIB orbifolds with similar properties. The resulting superpotential depends exponentially on the twisted sector Kähler (as opposed to complex structure) moduli. It does therefore not vanish on the sublocus in moduli space where the latter fields take a zero value. All this is in agreement with our interpretation of the deformation zero-modes as Goldstino modes in the model without discrete torsion: since the rigid divisors in presence of discrete torsion are stuck at 
the orbifold fixed loci, they do not probe a local higher symmetry except possibly the one preserved away from the orientifold fixed planes. There is therefore no rationale for them to exhibit Goldstino zero-modes other than the modes $\theta^{\alpha}$ and $\bar{\tau}^{\dot{\alpha}}$; the latter are absent as long as the instanton divisor intersects one of the orientifold fixed planes in a suitable manner, as before.

\section{$3.2 \mathcal{N}=2$ theories}

For $\mathcal{N}=2$ supersymmetry, the supersymmetric protected quantity is the prepotential and we are interested in theories where (1.4) holds. In this setting we can also sharply differentiate between the condition (1.4) holding over all the vector multiplet moduli space, or only on a sub-locus.

\subsubsection{Type II string theory on orbifolds}

Consider Type II string theory on $\mathbb{T}^{6}$ or $K 3 \times \mathbb{T}^{2}$, leading to $\mathcal{N}=8$ and $\mathcal{N}=4$ supersymmetry, respectively. Type II string theory on orbifolds of these spaces is an example where the prepotential is purely polynomial, in suitable coordinates, at least on a sub-locus of moduli space. To begin with, it is well known that the prepotential for $\mathcal{N}=8,4$ supersymmetric theories is purely cubic. However we now argue this is still true at least for some sub-loci of any orbifold of these spaces which breaks supersymmetry to $\mathcal{N}=2$.

To see this, note that the prepotential, which captures the low energy sector of vector multiplets in Type II string theory, is computed at string tree-level, i.e. at genus-zero on the string worldsheet. This is because vector multiplets and hypermultiplets decouple in $\mathcal{N}=2$ theories, and for Type II theories the coupling constant is given by the expectation value of a hypermultiplet and so we can take the weak coupling limit and compute the prepotential exactly by computing at string tree-level. Since the genus-zero amplitudes of the orbifold theory restricted to the untwisted vertex operators are identical to that of the unorbifolded theory (as there are no non-trivial cycles to include orbifold holonmy twists), we see that restricting the prepotential to the untwisted fields gives only a cubic polynomial as in the higher supersymmetric theory. If there are no massless fields in the twisted sectors this proves that the prepotential is exactly cubic for all moduli fields, because those all come from the untwisted sector. However, if there are massless fields in the twisted sectors, it is no longer true that including those moduli in the prepotential will lead to a cubic prepotential: this is because we now need to compute amplitudes involving vertex operators of twisted fields, which receive no protection from a higher supersymmetric theory, to which they do not belong. This means that in such a case, only if we set the expectation value of massless twisted states to zero are the amplitudes unmodified. Hence the prepotential is polynomial only on the sub-locus of vanishing twisted sector moduli.

Note that this argument critically uses the fact that the prepotential is generated at string tree-level in Type II string theory. For higher genus amplitudes, computing $F^{(g)}$ with $g>0$, it is no longer true that the relevant worldsheet has no one-cycles and so even the amplitudes involving untwisted vertex operators are no longer protected. An example of this is the correction to genus-one topological amplitudes discussed in [68]. As shown there, these amplitudes vanish for theories with $\mathcal{N}=8$ supersymmetry exemplified by Type 
II compactifications on $\mathbb{T}^{6}$. However they do not vanish on $\mathbb{T}^{4} / \mathbb{Z}_{2} \times \mathbb{T}^{2}=K 3 \times \mathbb{T}^{2}$ or $\mathbb{T}^{6} / \Gamma$ (for generic $\Gamma \subset \mathrm{SU}(3)$ ), which have $\mathcal{N}=4$ or $\mathcal{N}=2$ supersymmetry, even on the locus where twisted fields are set to zero.

Similarly, the argument does not imply the vanishing of the prepotential corrections e.g. for the heterotic string on a freely acting orbifold $\mathbb{T}^{6} / \Gamma$ because the prepotential receives corrections at one-loop level in the heterotic frame. Indeed, suppose we take a freely acting $\mathbb{Z}_{2}$ orbifold of $\mathbb{T}^{4} \times \mathbb{T}^{2}$ with the standard embedding, leading to gauge group $\left[E_{7} \times \mathrm{SU}(2)\right] \times$ $E_{8}$. At the massless level, the orbifold projects out, in particular, part of the states in the adjoint representation of the $E_{8}$ gauge factor. This leads to non-vanishing corrections to the prepotential at one-loop, in agreement with the above argument. We conclude that the appearance of an orbifold structure for an arbitrary string theory as such is not sufficient to guarantee the vanishing of the prepotential, be it everywhere in moduli space or only on a sub-locus. The same applies to $\mathcal{N}=1$ amplitudes. Thus the orbifold structure we have found is sufficient to guarantee protection against corrections only in specific cases, and is not sufficient for protection in a general situation.

\subsubsection{IIA string theory on the Enriques Calabi-Yau}

For further illustration, and also to make contact with the discussion in section 3.1, note that Type IIA string theory on the Enriques Calabi-Yau is an example of an $\mathcal{N}=2$ theory where the property (1.4) is satisfied over all the moduli space. ${ }^{14}$ The Calabi-Yau $X_{3}$ is the smooth resolution of the orbifold

$$
\theta:(0+, 0-, 0-), \quad \theta^{\prime}:\left(0-, \frac{1}{2}+, \frac{1}{2}-\right) .
$$

It is also possible to smooth out the first $\mathbb{Z}_{2}$ and write $X_{3}$ as a freely acting orbifold

$$
X_{3}=\frac{\mathbb{T}^{2} \times K 3}{\mathbb{Z}_{2}} .
$$

Because the orbifolding is freely acting, the Enriques Calabi-Yau has holonomy $\mathrm{SU}(2) \times \mathbb{Z}_{2}$ rather than $\mathrm{SU}(3)$. By the general argument of section 3.2.1, the worldsheet instanton corrections to the prepotential must be vanishing, and in fact in the entire moduli space because there exists no massless twisted sector since the involution is freely-acting.

Alternatively, we can understand the absence of prepotential corrections in a manner similar to the discussion in section 3.1 .1 by noting that every worldsheet instanton on $X_{3}$ necessarily has too many zero-modes to contribute to the prepotential. As in the $\mathcal{N}=1$ context, the presence of these universal zero-modes is related to supersymmetry. Technically it is a consequence of the fact that the Enriques Calabi-Yau is elliptically fibered over the Enriques surface $B_{2}$ with

$$
c_{1}\left(K_{B_{2}}\right) \in \operatorname{Tor} H^{2}\left(B_{2}, \mathbb{Z}\right)=\tilde{\Gamma}=\mathbb{Z}_{2} .
$$

\footnotetext{
${ }^{14}$ The first study of this Calabi-Yau was in [69]; in particular all genus-zero Gromov-Witten invariants vanish [70], which means that there are no worldsheet instanton corrections to the Type IIA prepotential. A closely related study of Type II string theory on this manifold has also appeared in [71].
} 
Note that this relation implies that also Tor $H^{2}\left(X_{3}, \mathbb{Z}\right)=\mathbb{Z}_{2}$. Duality between Type IIA on $X_{3}$ with base $B_{2}$ and F-theory on $B_{2} \times \mathbb{T}^{2}$ maps a worldsheet instanton on a curve $C$ on $B_{2}$ to a D3-brane along $C \times \mathbb{T}^{2}$. A D3-brane wrapping the curve $C$ in F-theory on $B_{2}$ gives rise to a string in six dimensions. Its worldsheet theory contains a collection of $4 h^{0}\left(C,\left.K_{B_{2}}\right|_{C}\right)=4$ universal zero-modes as well as $4 h^{0}\left(C,\left.K_{B_{2}}\right|_{C}\right)$ extra modes [72, 73], which we interpret as Goldstino modes. ${ }^{15}$ In reducing the string along $\mathbb{T}^{2}$ we obtain a worldsheet instanton in Type IIA string theory with corresponding zero-modes. These are the $\mathcal{N}=2$ analogue of the universal modes $\theta^{\alpha}$ and, respectively, the modes $\bar{\tau}^{\dot{\alpha}}$ in the $\mathcal{N}=1$ context. In particular there exist four additional zero-modes, analogous to the modes $\bar{\tau}$, whenever $\left.K_{B_{2}}\right|_{C}=\mathcal{O}_{C}$. For $X_{3}$ of the form (3.25) this is guaranteed for every curve $C \in B_{2}$ for the same reasons as in section 3.1.1.

\section{Characteristics of the examples with extra protection}

We have shown that there are a number of examples, and quite general constructions, which do not receive instanton corrections to their supersymmetric protected quantities, but preserve less than the expected amount of supersymmetry for such protection. It is natural to consider the general features of such examples, and how these may be responsible for this surprising amount of protection. In this section we will identify such general features.

Let us first introduce some notation. If in a supergravity theory a supersymmetric protected quantity, in the sense of the Introduction, vanishes, we say that the theory mimics a higher supersymmetric theory. For instance, if a theory with $\mathcal{N}=1$ supersymmetry has a vacuum with an exactly vanishing potential, it mimics an $\mathcal{N}=2$ theory.

Our claim is that all the examples constructed in this work, where a theory mimics one with higher supersymmetry, take the following form: there are two supergravity theories,

$$
\begin{array}{lll}
\text { Theory A : } & \mathcal{N}=k^{\prime} & \text { supersymmetric, } \\
\text { Theory B : } & \mathcal{N}=k<k^{\prime} & \text { supersymmetric } .
\end{array}
$$

Theory $A$ has an R-symmetry group containing a discrete subgroup $\Gamma$, which is necessarily gauged because there are no global symmetries in quantum gravity (in more than two spacetime dimensions). Theory $B$ is obtained as the orbifold quotient

$$
B=A / \Gamma
$$

As always in the context of (abelian) orbifolds (see for example [74]), the orbifold Theory $B$ exhibits a discrete gauge symmetry $\tilde{\Gamma}$ such that the neutral sector of $B$ with respect to $\tilde{\Gamma}$ coincides with the neutral sector of $A$ under $\Gamma .{ }^{16}$ Note, however, that $\tilde{\Gamma}$ is not an R-symmetry group of $B$. The neutral sector of Theory $B$ under symmetry $\tilde{\Gamma}$ is hence

\footnotetext{
${ }^{15}$ The first type of modes are the ones called $\mu_{+}$and $\tilde{\mu}_{+}$in table 3 of [73] in representation $(\mathbf{2}, 1)$ of $\mathbb{R}_{T}^{4}$ and the latter are the modes $\lambda_{-}$and $\tilde{\lambda}_{-}$in representation $(1,2)$. Here $\mathbb{R}_{T}^{4}$ refers to the four directions transverse to the string in $\mathbb{R}^{1,5}$.

${ }^{16}$ If $\Gamma$ is abelian, as in all examples considered in this paper, then $\Gamma$ and $\tilde{\Gamma}$ are isomorphic as groups.
} 
embeddable into Theory $A$, as the sector neutral under $\Gamma$. On the locus in the field space of $B$ where the symmetry $\tilde{\Gamma}$ is unbroken, the theory mimics aspects of a higher supersymmetric theory (more precisely the structure of an $\mathcal{N}=k^{\prime}$ supersymmetry as in Theory $A$ ).

The cases where Theory $B$ mimics the structure of higher supersymmetry over all of its moduli space, for example when the prepotential is cubic over all the vector multiplet moduli space, is a further restriction of this scenario. In such cases all the massless fields in Theory $B$ are neutral under $\tilde{\Gamma}$.

This structure is indeed realized in all the examples of theories mimicking higher supersymmetry in this paper. The sector of Theory $B$ charged under $\tilde{\Gamma}$ is identified, in orbifold language, with the twisted sector labeled by group elements of $\Gamma$. In the presence of such a twisted sector, e.g. for $\Gamma=\mathbb{Z}_{2}$, Theory $B$ has an exact $\tilde{\Gamma}=\mathbb{Z}_{2}$ symmetry which acts by sending all the twisted modes to minus themselves. Modding out the orbifolded theory by this gauged $\tilde{\Gamma}=\mathbb{Z}_{2}$ gives back the original theory. The sector of the orbifold theory invariant under $\tilde{\Gamma}$ comes from the untwisted sector of theory $A$, which was invariant under $\Gamma$.

The symmetry group $\tilde{\Gamma}$ expected in orbifold constructions can also be understood from geometric considerations in the cases without a massless twisted sector, where we have a freely acting quotient operating on Theory $A$ by a discrete symmetry $\Gamma$. Taking the quotient leads to a compactification manifold $M$ with non-trivial torsional group ${ }^{17}$ $\operatorname{Tor} H_{1}(M, \mathbb{Z})=\Gamma$. Non-trivial elements of this Tor $H_{1}$ precisely label the twisted sectors of the orbifold, i.e. the winding string states on these 1-cycles. $\tilde{\Gamma}$ is dual to this and can be viewed as the space of representations of $\Gamma$. Such torsional cohomology implies a discrete gauge symmetries in Theory $B[55,56]$ which in this case is nothing but the usual symmetry group associated to the existence of twisted sectors of the orbifold $M$ and their symmetry under interactions given by $\tilde{\Gamma}$ (see e.g. [75]).

\section{Discussion}

In this work we have argued that the superpotential and prepotential in a quantum gravity theory with four-dimensional $\mathcal{N}=1$ and $\mathcal{N}=2$ supersymmetry receive non-perturbative corrrections unless they are protected by supersymmetry, in the sense that the theory is related in a subtle way to a higher supersymmetric theory.

In all examples where we have observed this phenomenon, the relation to the higher supersymmetric theory is essentially via an orbifolding procedure. In absence of a twisted massless sector, the protection by this higher supersymmetry is at work all over moduli space, while more generally it can be broken if the massless twisted sector fields acquire a vacuum expectation value. In all examples, we have given various arguments for this behaviour. In the context of Type II compactifications with $\mathcal{N}=2$ supersymmetry, the absence of corrections to the prepotential could be explained by general properties of genuszero correlators in orbifolded theories. In other string theoretic examples, in particular in

\footnotetext{
${ }^{17}$ If the compactification underlying Theory $A$ has torsion itself, the freely acting quotient $a d d s$ the torsion factor $\Gamma$ to this independent torsional group.
} 
models with $\mathcal{N}=1$ supersymmetry, we have traced back the vanishing of the superpotential behaviour to the appearance of certain instanton zero-modes: these are to be interpreted as Goldstino modes associated with the spontaneous breaking of a higher supersymmetry probed locally by the instantons. The explicit breaking of the higher supersymmetry by the orbifolding is global in nature. As long as an instanton does not intersect any of the fixed loci of the orbifold, it probes the higher supersymmetry and therefore exhibits certain Goldstino modes in its worldvolume. The latter are lifted only via interactions involving the massless twisted sector fields, if present.

While in all the examples with a non-generic vanishing behaviour which we have found we have observed an orbifold structure, the latter does not in general guarantee a protection, for instance, of the superpotential or prepotential against corrections. We have exemplified this by recalling that e.g. the $\mathbb{T}^{6} / \mathbb{Z}_{2} \times \mathbb{Z}_{2}$ orbifold with discrete torsion [58] has a non-zero superpotential $[64,65,67]$ generated by instantons along blow-up divisors throughout its moduli space. This is in full agreement with our interpretation of the unliftable instanton zero-modes as Goldstino modes. Similarly in the $\mathcal{N}=2$ case, the heterotic string on a freely-acting orbifold can exhibit one-loop corrections to the prepotential, unlike our protected examples in the context of Type II theory, which are exact at genus zero.

Our results for theories with $\mathcal{N}=1$ and $\mathcal{N}=2$ theories in four dimensions prompt the question whether there can also be quantities in an $\mathcal{N}=0$ supersymmetric theory, such as the potential, which vanish if the theory is related to an $\mathcal{N}=1$ theory in a similar manner. Another candidate for a partly protected quantity arises in the context of the Weak Gravity Conjecture [76], which states that every gauge theory coupled to quantum gravity must exhibit some super-extremal particle of charge $(q, m)$ satisfying

$$
\frac{q^{2}}{m^{2}} \geq \frac{c}{M_{\mathrm{Pl}}^{d-2}} .
$$

Here the order one constant $c$ depends on the number of spacetime dimensions $d$ and the details of the theory such as presence of massless scalar fields [77-79]. According to a strong form of this conjecture [80], the equality should hold for a particle exactly including quantum corrections only if the theory is supersymmetric and the state is BPS: otherwise there is no protection against slight quantum corrections taking the particle away from the strict equality. In a sense this is similar to the general lesson of this paper that with lower supersymmetry everything that is allowed will happen. Taking this analogy further, even though we are not aware of any counterexamples to this conjecture, in the spirit of this paper one might wonder if there can exist exceptional non-supersymmetric theories in which nonetheless strict equality for the non-BPS WGC states holds, possibly again because of a subtle relation to a higher supersymmetric theory.

Note added. While this work was in completion we were notified of the work [81], which has some overlap with our ideas. 


\section{Acknowledgments}

We would like to thank Ralph Blumenhagen, Sergio Cecotti, Albrecht Klemm, Dieter Lüst and Stephan Stieberger for helpful discussions and correspondence. We have greatly benefited from the hospitality of UC Santa Barbara KITP where this project was completed.

The research of C.V. is supported in part by the NSF grant PHY-1719924 and by a grant from the Simons Foundation $(602883, \mathrm{CV})$. This research was supported in part by the National Science Foundation under Grant No. NSF PHY-1748958.

\section{A Flux lifting of D3-instanton zero-modes in F-theory}

In this appendix we discuss the lifting of D3-brane instanton zero-modes in F-theory by instanton flux. After briefly reviewing the general mechanism of [8] in appendix A.1, we argue in appendix A.2 that on $B_{3}=\mathbb{P}^{1} \times \mathbb{P}^{2}$ this mechanism does not lead to the generation of a superpotential by D3-brane instantons.

\section{A.1 General mechanism}

The possibility that instanton flux can lift some of the deformation modes of a D3-brane instanton in Type IIB/F-theory was pointed out in [8]. Consequently, the $\chi(\hat{D})=1$ condition is modified for instantons carrying instanton flux [8]. However, instanton flux can only lift (some of the) zero-modes counted by $H^{2}\left(D, \mathcal{O}_{D}\right)$ or $H^{2}\left(D,\left.K_{B_{3}}\right|_{D}\right)$ (see table 1 ), and not the other types of modes.

While in principle the flux lifting mechanism can be understood in full generality within F-theory [8] (see [9] for the related M-theory formulation), for practical purposes the simplest explanation is available in the weakly coupled Type IIB limit, provided it can be taken. Admissable instanton flux is then of the form [10]

$$
\mathcal{F} \in H_{-}^{1,1}(\tilde{D}) .
$$

In order for such flux to be able to lift some of the deformation modes, it must not lie in the space $\iota^{*} H_{-}^{1,1}\left(X_{3}\right)$ where $\iota: \tilde{D} \hookrightarrow X_{3}$ is the inclusion of the instanton divisor $\tilde{D}$ into $X_{3}$. Furthermore, the flux must be writable as [8]

$$
\mathcal{F}=\mathcal{F}_{0}+\sum_{a}\left(C_{a}-C_{a}^{\prime}\right)
$$

Here $\mathcal{F}_{0}$ is chosen such as to satisfy the Freed-Witten anomaly along $\tilde{D}$ and $C_{a}$ is a rigid curve on $X_{3}$ with orientifold image $C_{a}^{\prime}$. As long as $C_{a}-C_{a}^{\prime}$ is non-trivial as an element of $H_{-}^{1,1}(\tilde{D})$ such flux forces the divisor $\tilde{D}$ onto the locus where it contains the rigid curves $C_{a}$ and $C_{a}^{\prime}$, thereby lifting (some of) its deformation modes.

\section{A.2 No flux lifting for $B_{3}=\mathbb{P}^{1} \times \mathbb{P}^{2}$}

Consider now F-theory on an elliptic fibration $Y_{4}$ over the base $B_{3}=\mathbb{P}^{1} \times \mathbb{P}^{2}$. This is a special case of the more general class of base spaces given by a $\mathbb{P}^{1}$-fibration $p: B_{3} \rightarrow B_{2}$ (see the discussion around (B.1) in appendix B for more details). The trivial fibration $\mathbb{P}^{1} \times \mathbb{P}^{2}$ 
has a section $S_{-}$and we can furthermore consider the pullback $p^{*}(H)$ of the hyperplane class $H$ on $\mathbb{P}^{2}$ to $B_{3}$. The most general divisor on $B_{3}$ is hence of the form $D=a S_{-}+p^{*}(b H)$.

From the general expression for the arithmetic genus (2.6) one finds that for the pullback $\hat{D}$ of this divisor to $Y_{4}: 18$

$$
\chi\left(\hat{D}=\pi^{*} D\right)=-b^{2} \quad \text { for } \quad D=a S_{-}+p^{*}(b H) .
$$

According to the discussion in section 2.1.1, there are therefore no unfluxed instantons along divisors with $\chi(\hat{D})=1$ which would straightforwardly contribute to the superpotential [7].

It is important to note, however, that there are divisors whose additional zero-modes are deformation zero-modes, rather than Goldstino zero-modes counted by $h^{(1,0)}(\hat{D})$. In particular, for unfluxed instantons, one computes, for example,

$$
\begin{array}{ll}
h^{(i, 0)}\left(\hat{D}=\pi^{*} D\right)=(1,0,0,2) & \text { for } D=p^{*}(H) \\
h^{(i, 0)}\left(\hat{D}=\pi^{*} D\right)=(1,0,0,1) & \text { for } D=S_{-} .
\end{array}
$$

This raises the question if the additional deformation zero-modes counted by $h^{(3,0)}(\hat{D})$ for both types of divisors are lifted if the instanton carries suitable instanton flux, as in the example where $B_{3}=\mathbb{P}^{3}[8]$ reviewed in section 2.1.2. However, we now argue that this is not the case.

As for $S_{-}$, this follows already from the arguments given in [8]. In particular, deformation zero-modes cannot be lifted whenever the normal bundle exact sequence for the divisor $D$ on $B_{3}$ splits holomorphically in the sense that $\left.T_{B_{3}}\right|_{D}=T_{D} \oplus N_{D / B_{3}}$. This is indeed the case here because $D=S_{-}$is identified with the second factor in the direct product $B_{3}=\mathbb{P}^{1} \times \mathbb{P}^{2}$. To show that also the zero-modes of $D=p^{*}(H)$ cannot be lifted by instanton flux is more involved. Following [8] we approach this problem in the orientifold limit, which can always be taken for a generic Weierstrass model over $B_{3}$.

According to the general procedure [82], the Calabi-Yau 3-fold $X_{3}$ of the Type IIB orientifold is constructed as a double cover of $B_{3}$ given by a generic hypersurface in the toric ambient space $X_{4}$ shown in table 3 ,

$$
X_{3}: \quad \xi^{2}=P_{4,6}\left(z_{i}, u_{j}\right) .
$$

The orientifold action is

$$
s: \xi \rightarrow-\xi
$$

This space is a genus-one fibration over $\mathbb{P}^{2}$ with the genus-one fiber represented as a hypersurface in $\mathbb{P}_{1,1,2}$ with homogenous coordinates $\left[z_{1}: z_{2}: \xi\right]$. In particular, $z_{1}=0$ is the divisor associated with a bi-section, as analysed in detail in [83].

Consider first the double cover $\tilde{D}=\tilde{S}_{-}$of the divisor $D=S_{-}$on $B_{3}$. A generic member in this family is identified with the vanishing locus of the polynomial $c_{1} z_{1}+c_{2} z_{2}=0$. Such a divisor is a K3-surface, as is easily checked with the help of the adjunction formula. In

\footnotetext{
${ }^{18}$ This uses that for a smooth Weierstrass model with section $\sigma, c_{2}\left(Y_{4}\right)=12 \sigma \pi^{*} c_{1}\left(B_{3}\right)+11\left(\pi^{*} c_{1}\left(B_{3}\right)\right)^{2}+$ $\pi^{*} c_{2}\left(B_{3}\right)$. Together with the intersection numbers $S_{-} \cdot B_{3} S_{-}=-S_{-} \cdot B_{3} c_{1}(\mathcal{L})$ (cf. (B.4)) this implies $\chi(\hat{D})=-\frac{1}{2} \int_{B_{3}} D^{2} c_{1}\left(B_{3}\right)$.
} 


\begin{tabular}{|c|c|c|c|c|c|}
\hline$z_{1}$ & $z_{2}$ & $\xi$ & $u_{1}$ & $u_{2}$ & $u_{3}$ \\
\hline 1 & 1 & 2 & 0 & 0 & 0 \\
\hline 0 & $\mathrm{t}=0$ & 3 & 1 & 1 & 1 \\
\hline
\end{tabular}

Table 3. Scaling relations for toric ambient space $X_{4}$ of Calabi-Yau double cover $X_{3}$ associated with $B_{3}=\mathbb{P}^{1} \times \mathbb{P}^{2}$.

fact $X_{3}$ admits, in addition to the genus-one fibration, a K3-fibration over a rational curve, and $\tilde{S}_{-}$is precisely the fiber of this second type of fibration. This implies that $\tilde{S}_{-}$cannot contain any rigid curves. According to the discussion around (A.2) there exists therefore no instanton flux that can lift the deformation mode on $\tilde{S}_{-}$(and hence on $S_{-}$), in agreement with the general argument above.

The double cover $\tilde{D}=\tilde{p}^{*}(H)$ corresponds to a divisor $a_{1} u_{1}+a_{2} u_{2}+a_{3} u_{3}=0$ on $X_{3}$. Again we claim that there exists no instanton flux of the form (A.2) required to lift the two deformation modes. To see this, we need to understand the structure of rigid curves on the genus-one fibration $X_{3}$. Any holomorphic curve on $X_{3}$ is either a fibral curve, an curve on the base $B_{2}$ (i.e. lying in the section) or a linear combination. The base $B_{2}=\mathbb{P}^{2}$ does not contain any rigid curve. The general fiber is not rigid either. This leaves as the only source of rigidity fibral curves into which the genus-one fiber degenerates. Indeed, as analysed in [83], over isolated points $p_{a}$ on $B_{2}$, the genus-one fiber $F$ splits into two rigid homologous rational curves $C_{a}$ and $\tilde{C}_{a}, F \rightarrow C_{a}+\tilde{C}_{a}$ with $\left[C_{a}\right]=\left[\tilde{C}_{a}\right]=\frac{1}{2}[F]$ in $H_{2}\left(X_{3}, \mathbb{Z}\right)$. This happens over such points $p_{a}$ where the hypersurface equation (A.6) degenerates as

$$
\left(\xi+f_{a}\right)\left(\xi-f_{a}\right)=g_{a}^{2}
$$

for $f_{a}\left(z_{i}, u_{j}\right)$ and $g_{a}\left(z_{i}, u_{j}\right)$ suitable polynomials. The curves $C_{a}:\left(\xi+f_{a}\right)=g_{a}=0$ and $\tilde{C}_{a}\left(-\xi+f_{a}\right)=g_{a}=0$ are then homologous rational curves. We note that they map to one another under the orientifold involution (A.7), i.e. $C_{a}^{\prime}:=s\left(C_{a}\right)=\tilde{C}_{a}$.

Furthermore, $C_{a}$ and $C_{a}^{\prime}$ are homologous not only in $X_{3}$, but also within $\tilde{D}$. This is clear because the bi-section intersects $C_{a}$ and $C_{a}^{\prime}$ each in one point and the two intersection points are exchanged by a monodromy along a ramification divisor on $B_{2}$, corresponding to the existence of a chain connecting $C_{a}$ and $C_{a}^{\prime}$. In the present case, the ramification divisor is in the class $2 \bar{K}_{B_{2}}=6 H,{ }^{19}$ and since since this ramification locus of the bi-section always intersects the class $H$ on $\mathbb{P}^{2}$, it cannot be arranged for $C_{a}$ and $C_{a}^{\prime}$ to be not homologous on $\tilde{D}$. As a result, the zero-modes cannot be lifted by instanton flux of the form (A.2).

One can convince oneself that this problem persists for all other divisors on $B_{3}$. As a result, we have identified an example of an F-theory compactification with no divisors supporting fluxed or influxed instanton that can contribute to the superpotential without invoking other mechanisms of zero-mode lifting.

\footnotetext{
${ }^{19}$ In the notation of [84], see table 2.1 therein, the specific fibration corresponds to the choice $\beta=\bar{K}_{B_{2}}$, while from equ. (2.13) and table 2.2 one infers that the ramification divisor is in class $\left[c_{4}\right]=4 \bar{K}_{B_{2}}-2 \beta$.
} 


\section{B Stringy heterotic instantons}

In this appendix we study stringy NS5-brane instantons in the heterotic string. Their importance derives from the fact that they can in general induce a superpotential in heterotic string vacua even in situations where worldsheet instanton effects are known to lead to no such correction. In the sequel we provide what we believe is the first example where a stringy NS5-brane instanton is guaranteed to contribute to the superpotential.

While the zero-modes of NS5-brane instantons are difficult to study directly in the heterotic frame, they can in suitable settings be understood by duality. As shown in figure 1, heterotic-Type I duality maps NS5 instantons to D5 instantons in Type I, while under heterotic-Type IIB duality, NS5 instantons map to certain D3 instantons. The latter can sometimes be interpreted as gauge instantons, but in general can only be understood as stringy instantons.

In the sequel we will recall the dual description of heterotic NS5-brane instantons as D3-brane instantons in F-theory, first described in [7]. We will then provide an example where the latter instanton is guaranteed to contribute to the superpotential; this exemplifies that stringy NS5-brane instantons can indeed correct the superpotential even in absence of confining gauge symmetry or other types of gauge instantons in heterotic string theory.

The heterotic string compactified on an elliptically fibered Calabi-Yau 3-fold $Z_{3}$ with base $B_{2}$ is dual to F-theory on an elliptic 4 -fold $Y_{4}$ whose base $B_{3}$ admits a fibration [85]

$$
\begin{aligned}
p: \quad \mathbb{P}^{1} \rightarrow & B_{3} \\
& \downarrow \\
& B_{2}
\end{aligned}
$$

Such $B_{3}$ can be expressed as the projectivised bundle

$$
B_{3}=\mathbb{P}\left(\mathcal{O}_{B_{2}} \oplus \mathcal{L}\right),
$$

where the line bundle $\mathcal{L}$ on $B_{2}$ describes the twist of the $\mathbb{P}^{1}$-fibration. There are two distinguished sections, whose associated divisor classes $S_{-}$and $S_{+}$are given by

$$
S_{-}=c_{1}(\mathcal{O}(1)), \quad S_{+}=S_{-}+c_{1}(\mathcal{L}),
$$

and which satisfy

$$
S_{-} \cdot B_{3} S_{+}=0
$$

Here $\mathcal{O}(1)$ denotes the line bundle on $B_{3}$ whose restriction to the $\mathbb{P}^{1}$-fiber is the line bundle $\mathcal{O}(1)$. In terms of these quantities,

$$
c_{1}\left(B_{3}\right)=2 S_{-}+p^{*}\left(c_{1}(\mathcal{L})+c_{1}\left(B_{2}\right)\right) .
$$

The heterotic gauge group in the two $E_{8}$ factors maps to the gauge groups of the 7-brane stacks localised along $S_{-}$and $S_{+}$, respectively. For more details we refer e.g. to [86] and references therein. 
Consider an M5-brane instanton on $Y_{4}$ wrapping a divisor $\hat{D}=\pi^{*}(D)$ for $D$ a divisor on $B_{3}$. Such instantons dualize to the following objects in the heterotic frame [7]:

$$
\begin{aligned}
& D=S_{ \pm} \\
& D=p^{*}(C)
\end{aligned} \Longleftrightarrow \begin{aligned}
& \text { NS5-brane instanton on } Z_{3} \\
& \text { worldsheet-instanton on } C \subset B_{2}
\end{aligned}
$$

Here $C$ is a curve on $B_{2}$. An instanton along a general divisor in class $D=a S_{-}+p^{*}(C)$ then maps to a bound state of an NS5-worldsheet instanton. Note that for all types of instantons one must again sum over all possible instanton flux configurations. For a recent systematic study of D3-brane instantons in the context of F-theory/heterotic duality, see also [87].

After this review we now exemplify that stringy NS5-brane instantons can indeed contribute to the superpotential. For the purpose of providing such an example it suffices to specialise to $B_{2}=\mathbb{P}^{2}$. The possible choices of twist bundle are simply $\mathcal{L}=\mathcal{O}_{\mathbb{P}^{2}}(t H)$ with $H$ the hyperplane class of $\mathbb{P}^{2}$. As analysed in [86], for $t \geq 4$, the theory contains a nonHiggsable gauge group along the section $S_{-}$. In particular, this invalides the expression in the fourth column of table 1 , which assumed that $\hat{D}$ is a smooth divisor. For $t=0,1,2,3$, on the other hand, we can take the M-theory Calabi-Yau 4-fold $Y_{4}$ to be a smooth Weierstrass model over $B_{3}$, leading to a $4 \mathrm{~d} N=1$ theory with trivial gauge group. For simplicity we focus on such smooth situations. For unfluxed instantons along $S_{\mp}$, evaluating the multiplicities in table 1 gives $^{20}$

$$
\begin{array}{lll}
t=0: & h^{(i, 0)}\left(\pi^{*} S_{-}\right)=(1,0,0,1), & h^{(i, 0)}\left(\pi^{*} S_{+}\right)=(1,0,0,1) \\
t=1,2: & h^{(i, 0)}\left(\pi^{*} S_{-}\right)=(1,0,0,0), & h^{(i, 0)}\left(\pi^{*} S_{+}\right)=(1,0,0, a) \\
t=3: & h^{(i, 0)}\left(\pi^{*} S_{-}\right)=(1, b, 0,0), &
\end{array}
$$

for

$$
a=\frac{1}{2}(t+2)(t+1), \quad b=\frac{1}{2}(t-1)(t-2) .
$$

For $t=1,2$, we conclude that an unfluxed NS5-brane instanton in the heterotic theory dual to the instanton along $S_{-}$straightforwardly contributes to the superpotential, even without invoking other effects. For $t=0$ and $t=3$, at least one of the unfluxed instantons along $S_{-}$or $S_{+}$has only extra deformation modes in $H^{3,0}(\hat{D}) \cdot{ }^{21}$ Such zero-modes are in principle amenable to lifting by instanton flux [8], though whether or not this is possible depends on the intricate details of the geometry, and to the lifting mechanism involving spacetime filling D3-branes studied in section 2.1.3.

\section{Superpotentials in heterotic compactifications}

In this section we study superpotentials in the heterotic string. The $E_{8} \times E_{8}$ or $\mathrm{SO}(32)$ heterotic string on a Calabi-Yau threefold gives an $\mathcal{N}=1$ supergravity in four dimensions. Since the heterotic string in ten dimensions includes a non-Abelian gauge sector,

\footnotetext{
${ }^{20}$ Eq. (B.5) and (B.4) imply that $\left.K_{B_{3}}\right|_{S_{-}} \simeq \mathcal{O}_{\mathbb{P}^{2}}(t-3)$ and $\left.K_{B_{3}}\right|_{S_{+}} \simeq \mathcal{O}_{\mathbb{P}^{2}}(-t-3)$. The result then follows from Bott's theorem for line bundle cohomologies on $\mathbb{P}^{2}$.

${ }^{21}$ The statement for $t=0$ holds for any base $B_{2}$ and trivial twisting $c_{1}(\mathcal{L})=0$.
} 
before worrying about instanton contributions to the superpotential we should consider gauge theoretic contributions such as gaugino condensation. ${ }^{22}$ With regards to instantons, there are three types of instantons which can potentially contribute to the superpotential: worldsheet instantons, NS5 gauge instantons, and NS5 stringy instantons. ${ }^{23}$

NS5 stringy instantons are difficult to study explicitly in the heterotic context. As exemplified in appendix B they can in general contribute to the superpotential as stringy instantons. Unless this can be ruled out in specific settings, this possibility therefore generally invalidates any candidate for a theory with $W=0$.

The aim of this appendix is to determine whether it is possible to show that there are no heterotic compactifications with vanishing superpotential even if we leave aside such stringy NS5 brane instantons. We will not arrive at a definitive conclusion, due to recent developments in [36], but will present a serious contender for a setup with no gauge or worldsheet instanton contributions. We therefore believe that a claim for the universal generation of a superpotential in generic heterotic compactifications must involve stringy NS5 instantons.

Before proceeding let us note that Type I vacua are S-dual to heterotic vacua and so are in this sense also covered by the analysis of this section. See, however, e.g. [88-90] for studies of instantons directly in a Type I setting.

Heterotic worldsheet instantons are dual to certain D3 instantons as reviewed in appendix B. While they generically generate a superpotential, there are some special circumstances where this can be shown not to occur [31-33] (see [34-37] for recent work). It was proposed that worldsheet instantons are absent on favourable complete-intersection Calabi-Yau manifolds embedded in weighted projective spaces or toric spaces, and for which the bundle is inherited from the ambient space [33, 34]. This Beasley-Witten (BW) theorem is based on earlier work in [32] which showed that worldsheet instantons do not destabilise certain vacua which have a $(0,2)$ sigma model description. The argument relies on compactifying the moduli space as in the Riemann sphere. The superpotential is then a holomorphic function on the compact moduli space, and it has a universal zero at the decompactification limit. It must therefore either be exactly vanishing or have somewhere a pole. To determine this one needs to know the full moduli space, which includes small volumes away from the supergravity regime. A sigma-model description allows for this, and can be used to argue for the absence of poles. Recently, in [36, 37] it was shown that many compactifications which apparently satisfy the requirements for the BW theorem in fact do have non-vanishing worldsheet instantons. This was attributed to the non-compactness of the bundle moduli space, and therefore suggests that actually this must be imposed as a further independent criterion which is difficult to check. If there is a sigma model description of the setting, then in [91] a prescription for checking this compactness was given.

Let us now discuss the consequences of the Beasley-Witten theorem for standard embeddings and for non-standard embeddings in turn.

\footnotetext{
${ }^{22}$ In some sense, this applies also to Type II string theories, since introducing orientifold planes to break the supersymmetry to $\mathcal{N}=1$ requires also introducing branes to cancel the tadpoles.

${ }^{23}$ In the presence of NS5 spacetime-filling branes, there are also E-string instantons.
} 


\section{C.1 Standard embeddings}

In the standard embedding the gauge bundle is identified with the tangent bundle of the Calabi-Yau. For favorable Calabi-Yau spaces, the gauge bundle is therefore inherited from the ambient space, and the standard embedding automatically satisfies the conditions for the Beasley-Witten theorem to hold. Furthermore, unlike for more general gauge bundles, the standard embedding does not require the introduction of spacetime-filling NS5-branes. These objects are dual to D3-branes in F-theory which, as argued in section 2.1.3, affect the dynamics of instantons in a non-trivial way. Both these points make this class of constructions an interesting testing ground for the generation of a superpotential. Prior to discussing the worldsheet instantons, however, we must analyze the potential generation of a superpotential by strong gauge dynamics.

The results of the following discussion can be summarized as follows:

1. For the standard embedding, there is always a gaugino condensate generating a superpotential $W_{\text {gauge }}$. This superpotential alone may or may not allow for a solution to $W_{\text {gauge }}=d W_{\text {gauge }}=0$, even though genericity arguments would suggest this not to occur except on special backgrounds.

2. If $\chi\left(Z_{3}\right) \neq 0$ with $Z_{3}$ the heterotic Calabi-Yau, the Beasley-Witten vanishing theorem for the superpotential due to worldsheet instantons is a priori not valid even on favorable manifolds, while we are not aware of favorable examples for $\chi\left(Z_{3}\right)=0$ which do not lead to $\mathcal{N} \geq 2$ supersymmetry.

To see the first point, recall that in the $E_{8} \times E_{8}$ heterotic string the gauge bundle is completely embedded into only one of the $E_{8}$ factors. This results in a non-perturbative potential induced through gaugino condensation in the other $E_{8}$ factor, which has no massless charged matter. The standard embedding for the $\mathrm{SO}(32)$ (or more precisely $\left.\operatorname{Spin}(32) / \mathbb{Z}_{2}\right)$ heterotic string breaks the gauge group as

$$
\mathrm{SO}(32) \rightarrow \mathrm{SO}(26) \times \mathrm{U}(1) .
$$

This leads to charged chiral matter under the $\mathrm{SO}(26)$ non-Abelian factor which can prevent gaugino condensation. The chirality of the charged matter in representation $\mathbf{R}$, denoted $\chi(\mathbf{R})$, is set by the Euler number of the Calabi-Yau $\chi\left(Z_{3}\right)$,

$$
\begin{aligned}
\chi\left((\mathbf{2 6})_{1}\right) & \equiv \# \mathbf{2 6}_{1}-\# \overline{\mathbf{2 6}}_{1}=\chi\left(Z_{3}\right), \\
\chi\left((\mathbf{1})_{2}\right) & \equiv \# \mathbf{1}_{2}-\# \mathbf{1}_{-2}=-\chi\left(Z_{3}\right),
\end{aligned}
$$

where the subscript denotes the $\mathrm{U}(1)$ charge. The chiral matter is protected and hence massless, and therefore for sufficiently large $\chi\left(Z_{3}\right)$ will prevent gaugino condensation in the infrared. However, there is also a 1-loop D-term which takes the form (see, for example [92])

$$
D_{\chi} \sim \sum_{i}\left(\left|\phi_{i}\right|^{2}-\left|\tilde{\phi}_{i}\right|^{2}\right)+2\left(\sum_{j}\left|\psi_{j}\right|^{2}-\left|\tilde{\psi}_{j}\right|^{2}\right)+\frac{c}{2} g_{s}^{2} \chi\left(Z_{3}\right)
$$

where $\phi_{i}$ and $\psi_{j}$ denote the complex scalars in the $\mathcal{N}=1$ chiral multiplets in representation $(\mathbf{2 6})_{1}$ and $(\mathbf{1})_{2}$ and $\tilde{\phi}_{i}$ and $\tilde{\psi}_{j}$ the complex scalars in representation $(\mathbf{2 6})_{-1}$ and $(\mathbf{1})_{-2}$. The 
constant $c$ is of order one, and $g_{s}$ is the string coupling. This D-term implies that the $\mathrm{U}(1)$ must be broken in the vacuum precisely when $\chi\left(Z_{3}\right) \neq 0$, i.e. when there is potentially no gaugino condensation. The breaking of the $\mathrm{U}(1)$ then removes the net chirality. From the field theory perspective we can look at the superpotential operators

$$
W \supset \mathbf{1}_{-2} \mathbf{2 6}_{1} 2 \mathbf{6}_{1}+\text { c.c. . }
$$

From (C.3) we see that if, say, $\chi\left(Z_{3}\right)>0$, then we need to give an expectation value to the $(\mathbf{1})_{-2}$ fields which gives a mass to any chiral net number of $\mathbf{2 6}_{1}$ fields. That there always exists an appropriate $(\mathbf{1})_{-2}$ field to give an expectation value to was shown in [93] for a wide class of models, and conjectured to hold generally in [94]. The resulting vacuum then restores supersymmetry at the perturbation theory level (see, for example [95, 96] for recent studies). Note that the superpotential (C.4) also shows that there is an obstruction to solving the D-term condition by giving an expectation value to the $\mathbf{2 6}_{1}$ fields and thereby also breaking the non-Abelian gauge group.

We conclude that no matter the value of $\chi\left(Z_{3}\right)$, the spectrum of the standard embedding for the $\mathrm{SO}(32)$ heterotic string is non-chiral with a non-Abelian gauge group. This means that at a generic point in moduli space all the matter will be massive, leading to a non-perturbative superpotential in the infrared. More precisely, there exists a non-perturbative superpotential with a pre-factor which is moduli dependent and vanishes on certain special sub-loci of the moduli space where a sufficient number of vector-like charged states are massless. If we denote the moduli fields (bundle and complex-structure) collectively as $u^{i}$, then the superpotential takes the schematic form

$$
W_{\text {gauge }} \sim f\left(u^{i}\right) e^{-\frac{a}{g_{s}}} .
$$

Here $a$ is some constant and $f$ is a function of the moduli which vanishes on certain special loci denoted $u^{i}=u_{0}^{i}$, i.e. $f\left(u_{0}^{i}\right)=0$. We cannot exclude in full generality that on the locus $u_{0}^{i}$ also $d W_{\text {gauge }}=0$, though based on genericity arguments alone this is not expected; in any event, $W_{\text {gauge }}$ does not include the remaining contributions to the superpotential, especially from stringy NS5-brane instantons. ${ }^{24}$

Having studied the effect of gaugino condensation we can now turn to the generation of a superpotential via worldsheet instantons. In the case $\chi\left(Z_{3}\right) \neq 0$ we have just seen that the D-term requires an expectation value for a charged field. This corresponds to a deformation of the gauge bundle, and the deformed bundle is no longer expected to be inherited from the ambient space. Therefore, the BW vanishing theorem will not hold in general. In the case $\chi\left(Z_{3}\right)=0$, the $\mathrm{BW}$ theorem should hold at least as long as the manifold is a favourable embedding in a toric space.

Let us consider the Complete Intersection Calabi-Yau manifolds (CICYs) as a sample set. There are 52 cases with $\chi\left(Z_{3}\right)=0$. They are composed of 22 cases of direct product manifolds with a torus (which have enhanced supersymmetry), 15 cases with $h^{1,1}=19$ which are representations of the Schoen manifold, and 15 cases with $h^{1,1}=15$, which

\footnotetext{
${ }^{24}$ This is in notable difference to the non-generic setting in section 3.1.2, where we do argue for the existence of a sub-locus in moduli space with $W=d W=0$.
} 
are also the same manifold. The two non-product manifolds are not favourable when embedded in the projective spaces. ${ }^{25}$ Interestingly, this leaves as the only class of CICYs with $\chi\left(Z_{3}\right)=0$ those which lead to enhanced supersymmetry, which are therefore not of relevance when it comes to studying the generation of a superpotential.

\section{C.2 Non-standard embeddings}

We have accumulated evidence (though not provided a definitive proof) that standard embeddings for the heterotic string lead to the generation of a superpotential. It is also possible to consider bundles which are not the standard embedding. Indeed, the largest set of realistic heterotic models in the geometric regime are constructed this way [98-100]. When studying non-standard embeddings it is crucial for our purposes to consider the Bianchi identity, or tadpole condition, which takes the schematic form

$$
\operatorname{Tr}(F \wedge F)-\operatorname{Tr}(R \wedge R)=\delta_{\mathrm{NS} 5} .
$$

The first term is the contribution from the second Chern class of the bundle, the second term from the curvature, and if these are not equal then they must be balanced by the inclusion of spacetime filling NS5-branes. In this case it is no longer clear that the BW vanishing theorem holds. Indeed, dualising the results of section 2.1.3 to the heterotic string suggests that there are new potential instanton contributions in the presence of such spacetime-filling NS5 branes. The best controlled candidates for theories with $W=0$ are therefore embeddings which saturate the Bianchi identity and so do not require any NS5 branes.

The most natural way to saturate the Bianchi identity (C.6) in the $E_{8} \times E_{8}$ heterotic string is through the introduction of a further bundle on the hidden $E_{8}$. We will consider two types of models which will illustrate two aspects of the vacuum stability.

The first model is based on the case studied in [101]. ${ }^{26}$ This is a compactification utilising line bundles, based on the earlier work [98-100, 102]. There are line bundles on both of the $E_{8}$ factors, such that the Bianchi identity is saturated and there is no need to introduce NS5 branes. The resulting gauge group is $\mathrm{SU}(5) \times \mathrm{SU}(4) \times \mathrm{U}(1)^{9}$, and the matter spectrum is such that there is no gaugino condensation or gauge instantons in the non-Abelian factors. This is a good testing ground to see if the D-terms require breaking the U(1) symmetries in such vacuum. While in [101] the D-terms were solved with singlet expectation values which break the $\mathrm{U}(1) \mathrm{s}$, this need not be the case in general. Indeed, we find the following solution for the divisor volumes $\mathcal{V}_{i}$, with $i=1,2,3,4$,

$$
\mathcal{V}_{2}=\mathcal{V}_{1}+6 X, \quad \mathcal{V}_{3}=\mathcal{V}_{1}+2 X, \mathcal{V}_{4}=\mathcal{V}_{1}+8 X
$$

Here $X$ denotes the 1-loop contribution to the D-term, as in the last term of (C.3). The important point is that the 1-loop contribution is cancelled by the relative differences in

\footnotetext{
${ }^{25}$ However, in [97] it was argued that one can utilise an ineffective splitting to rewrite the Schoen manifold as a favourable embedding in a certain del Pezzo space, and the second manifold as a favourable embedding in projective spaces. Then an interesting question is whether the BW theorem can hold when the manifolds are rewritten this way. One piece of evidence against it could be that the Schoen manifold does support bundles which admit worldsheet instanton corrections [30].

${ }^{26}$ We restrict to the case $k=0$ in the notation of [101].
} 
the volumes of the divisors, so the latter can remain large and in the perturbative regime. This proves that there are examples where there are no worldsheet instantons, no gaugino condensation in the infrared, and the U(1)s need not be broken by the D-terms. There is still a subtlety in this model however, because if appropriate F-flat directions exist in the charged singlet moduli space, then it is possible (even if not enforced by the D-terms) to break all the $\mathrm{U}(1) \mathrm{s}$ and make the spectrum non-chiral with respect to the $\mathrm{SU}(4)$. This would then imply a gaugino condensation contribution to the superpotential which vanishes on the split locus where the charged singlets have vanishing expectation values. However, this does not guarantee that this locus is the minimum of the potential, and so the vacuum may still be destabilised due to gaugino condensation.

To make things even sharper we can consider a second model which is purely nonAbelian. We consider compactifications of the $E_{8} \times E_{8}$ heterotic string with monad bundles studied in [103]. First note that monad bundles are expected to satisfy the BW vanishing theorem, at least on favourable Calabi-Yau manifolds [33]. We will consider the quintic, which is favourable, and so there should not be any worldsheet instanton corrections. From table 4 in [103] we see that there exist two SU(3) monad bundles with second Chern classes of 3 and 7 (relative to the square of the overall Kähler form). Together with $c_{2}\left(Z_{3}\right)=10$ for the quintic, herefore taking one such bundle on each $E_{8}$ factor saturates the Bianchi identity and there are no NS5 branes. The resulting theory has gauge group $E_{6} \times E_{6}$, with 60 chiral 27s under the first $E_{6}$ and 15 under the second. On general grounds it is expected that there is no gaugino condensation for number of $27 \mathrm{~s}$ larger than 2 , which is easily satisfied for both the $E_{6}$ factors. There are no U(1)s, and so no D-terms, and it is not possible to lift the chiral matter without also breaking the non-Abelian gauge group.

This model suggests, as claimed at the start of this appendix, that the only potential universal obstruction to compactifications with $W=0$ in the heterotic string must come from stringy NS5 instantons. The only subtlety is the one discussed in the recent work $[36,37]$, which showed that sometimes the BW theorem does not hold due to noncompactness of the moduli space. It would be interesting to apply the more refined criteria of $[36,37]$ to this, and other similar, examples.

\section{Type IIA, Type I and M-theory vacua}

Type IIA compactifications on Calabi-Yau orientifolds, and M-theory compactifications on $G_{2}$ manifolds, lead to $\mathcal{N}=1$ four-dimensional theories. In these cases the geometry associated to the relevant instantons is real, rather than holomorphic, specifically special Lagrangian 3-cycles for IIA D2-brane instantons and associative manifolds for membrane instantons on $G_{2}$ manifolds. It is difficult to study such real submanifolds explicitly, and in particular to determine the associated zero-modes. Things are complicated further by an incomplete understanding of the effects of worldvolume fluxes in Type IIA, and the dual higher derivative effects in M-theory.

Type IIA vacua can be grouped into the F-theory vacua of section 2.1 since they are related through mirror symmetry. This is only the case for one of two types of orientifold projections in IIA, the other case being mapped to a Type I mirror. The Type I vacua are 
dual to heterotic $\mathrm{SO}(32)$ vacua. For the standard embedding of the gauge bundle in such vacua, we argue in appendix $\mathrm{C}$ that there is a non-perturbative superpotential induced due to gaugino condensation. The superpotential is therefore not vanishing everywhere in field space, and it is difficult to determine if there is a minimum where the superpotential vanishes. More general gauge bundle choices, different from the standard embedding, are difficult to study at the non-perturbative level, especially in the presence of spacetimefilling NS5-branes which can affect the worldsheet instanton corrections on the heterotic side (and which map to D1 instantons in Type I). Overall, we do not find the Type IIA and Type I settings offer sufficiently good control to search for examples with vanishing corrections.

The case of M-theory on $G_{2}$ manifolds, which yields an $\mathcal{N}=1$ supergravity [104], receives potential instanton contributions to the superpotential from M2 branes on associative 3-cycles. Since the microscopic theory for M2 branes is not known, it is not possible to calculate the condition for contributing to the superpotential explicitly as in Type II. But it is natural to perform an analysis similar to [7] in terms of deformation modes of the cycle. This was performed in [105] which led to the proposal that M2 instantons contribute to the superpotential if they wrap rigid associative 3-cycles. See also [106] for similar early work. The topic of instantons on $G_{2}$ manifolds is actively studied, boosted by the new constructions of $G_{2}$ manifolds using Twisted Connected Sums (TCS) [107-109] (see [110] for the earlier resolved orbifold constructions). We refer, in particular, to [111] for studies of associative cycles on such manifolds, as well as [112-118] for more general studies of associative cycles and instantons. The construction of TCS $G_{2}$ manifolds lends itself nicely to duality with heterotic and Type II string theories, which has been studied in [30, 119-122].

Studying instantons in M-theory has some unique elements. For example, because all the massless fields have axionic components, the Pfaffian prefactor of the instanton must be either a constant or a modular function of the fields. There are few known cases of the construction of such modular functions for $G_{2}$ manifolds, one example being in [30]. This example, the so-called $E_{8}\left(\times E_{8}\right)$ superpotential, also has duals in the heterotic string and in F-theory, and so is discussed in section 2.1. Another unique aspect is that all the instantons come from a single type of brane, the M2, which also does not support worldvolume fluxes. This would suggest that $G_{2}$ manifolds can be good examples of vacua which receive no corrections if the $G_{2}$ manifold supports no rigid associative cycles. However, there are no such known examples (to our knowledge). ${ }^{27}$ More generally, the analogues of effects that can lift zero-modes in Type II constructions, such as fluxes, are poorly understood in the $G_{2}$ setting. Possibly this can be attributed to higher fermions operators or higher derivative terms, such as the quartic fermionic terms analyzed in [33].

It is worth noting that mathematically, $G_{2}$ vacua which have no instanton corrections are denoted as unobstructed $G_{2}$ manifolds in [118]. There are simple examples of unobstructed $G_{2}$ manifolds given in [118], but these actually preserve $\mathcal{N}=2$ supersymmetry.

\footnotetext{
${ }^{27}$ The closest constructions we found are the TCS geometries in [111] (table 5) which have no rigid associative cycles that are constructed through a particular methodology, leaving open the possibility that other constructions of associative cycles on these manifolds might be possible.
} 
Flux vacua. Turning on background closed-string fluxes will in general induce a classical superpotential [23]. However, there may still exist vacua where this superpotential vanishes. For example, in Type IIB string theory there are points in complex-structure moduli space where the flux superpotential vanishes [123-125]. However, this does not directly affect the presence or absence of instantons, which in this case would be in the Kähler moduli. If anything, Type IIB background fluxes might lift D3-brane instanton zero-modes (see [26] for the state of the art and further references), allowing them to contribute to the superpotential.

One aspect of fluxes which does forbid certain instantons are anomalies induced on the D-brane worldvolume. For example, in Type IIA string theory turning on NS H-flux through a 3-cycle forbids any D2 instantons to occur on that cycle through a Freed-Witten anomaly. This can also be understood from a supergravity perspective in terms of the flux gauging an isometry in the field space which must be an exact flat direction [126]. However, there is no known example where there is a vacuum of vanishing superpotential where all the instantons are projected out this way. ${ }^{28}$

Open Access. This article is distributed under the terms of the Creative Commons Attribution License (CC-BY 4.0), which permits any use, distribution and reproduction in any medium, provided the original author(s) and source are credited.

\section{References}

[1] C. Vafa, The String landscape and the swampland, hep-th/0509212 [INSPIRE].

[2] T.D. Brennan, F. Carta and C. Vafa, The String Landscape, the Swampland and the Missing Corner, PoS(TASI2017)015 [arXiv:1711.00864] [inSPIRE].

[3] E. Palti, The Swampland: Introduction and Review, Fortsch. Phys. 67 (2019) 1900037 [arXiv: 1903.06239] [INSPIRE].

[4] E. Witten, Small instantons in string theory, Nucl. Phys. B 460 (1996) 541 [hep-th/9511030] [INSPIRE].

[5] M. Bianchi, S. Kovacs and G. Rossi, Instantons and Supersymmetry, Lect. Notes Phys. 737 (2008) 303 [hep-th/0703142] [INSPIRE].

[6] R. Blumenhagen, M. Cvetič, S. Kachru and T. Weigand, D-Brane Instantons in Type II Orientifolds, Ann. Rev. Nucl. Part. Sci. 59 (2009) 269 [arXiv:0902.3251] [INSPIRE].

[7] E. Witten, Nonperturbative superpotentials in string theory, Nucl. Phys. B 474 (1996) 343 [hep-th/9604030] [INSPIRE].

[8] M. Bianchi, A. Collinucci and L. Martucci, Magnetized E3-brane instantons in F-theory, JHEP 12 (2011) 045 [arXiv:1107.3732] [INSPIRE].

[9] M. Kerstan and T. Weigand, Fluxed M5-instantons in F-theory, Nucl. Phys. B 864 (2012) 597 [arXiv: 1205.4720] [INSPIRE].

\footnotetext{
${ }^{28}$ More generally, this is part of an interaction between instantons and twisted K-theory, see for example [3] for a discussion of this.
} 
[10] T.W. Grimm, M. Kerstan, E. Palti and T. Weigand, On Fluxed Instantons and Moduli Stabilisation in IIB Orientifolds and F-theory, Phys. Rev. D 84 (2011) 066001 [arXiv:1105.3193] [INSPIRE].

[11] O.J. Ganor, A Note on zeros of superpotentials in F-theory, Nucl. Phys. B 499 (1997) 55 [hep-th/9612077] [INSPIRE].

[12] R. Blumenhagen, M. Cvetič and T. Weigand, Spacetime instanton corrections in $4 D$ string vacua: The Seesaw mechanism for D-brane models, Nucl. Phys. B 771 (2007) 113 [hep-th/0609191] [INSPIRE].

[13] L.E. Ibáñez and A.M. Uranga, Neutrino Majorana Masses from String Theory Instanton Effects, JHEP 03 (2007) 052 [hep-th/0609213] [INSPIRE].

[14] B. Florea, S. Kachru, J. McGreevy and N. Saulina, Stringy Instantons and Quiver Gauge Theories, JHEP 05 (2007) 024 [hep-th/0610003] [INSPIRE].

[15] M. Haack, D. Krefl, D. Lüst, A. Van Proeyen and M. Zagermann, Gaugino Condensates and D-terms from D7-branes, JHEP 01 (2007) 078 [hep-th/0609211] [InSPIRE].

[16] A. Klemm, B. Lian, S.S. Roan and S.-T. Yau, Calabi-Yau fourfolds for M-theory and F-theory compactifications, Nucl. Phys. B 518 (1998) 515 [hep-th/9701023] [INSPIRE].

[17] R. Blumenhagen, A. Collinucci and B. Jurke, On Instanton Effects in F-theory, JHEP 08 (2010) 079 [arXiv: 1002.1894] [InSPIRE].

[18] A. Grassi, Divisors on elliptic Calabi-Yau four folds and the superpotential in F-theory. 1. J. Geom. Phys. 28 (1998) 289 [alg-geom/9704008] [InSPIRE].

[19] A. Grassi, On minimal models of elliptic threefolds, Math. Ann. 290 (1991) 287.

[20] M. Cvetič, R. Richter and T. Weigand, Computation of D-brane instanton induced superpotential couplings: Majorana masses from string theory, Phys. Rev. D 76 (2007) 086002 [hep-th/0703028] [INSPIRE].

[21] S. Sethi, C. Vafa and E. Witten, Constraints on low dimensional string compactifications, Nucl. Phys. B 480 (1996) 213 [hep-th/9606122] [INSPIRE].

[22] J. Gray, A.S. Haupt and A. Lukas, All Complete Intersection Calabi-Yau Four-Folds, JHEP 07 (2013) 070 [arXiv: 1303.1832] [INSPIRE].

[23] S. Gukov, C. Vafa and E. Witten, CFT's from Calabi-Yau four folds, Nucl. Phys. B 584 (2000) 69 [Erratum ibid. 608 (2001) ] [hep-th/9906070] [INSPIRE].

[24] R. Kallosh, A.-K. Kashani-Poor and A. Tomasiello, Counting fermionic zero modes on M5 with fluxes, JHEP 06 (2005) 069 [hep-th/0503138] [INSPIRE].

[25] M. Billó, L. Ferro, M. Frau, F. Fucito, A. Lerda and J.F. Morales, Flux interactions on D-branes and instantons, JHEP 10 (2008) 112 [arXiv:0807.1666] [INSPIRE].

[26] M. Bianchi, G. Inverso and L. Martucci, Brane instantons and fluxes in F-theory, JHEP $\mathbf{0 7}$ (2013) 037 [arXiv: 1212.0024] [INSPIRE].

[27] F. Marchesano and L. Martucci, Non-perturbative effects on seven-brane Yukawa couplings, Phys. Rev. Lett. 104 (2010) 231601 [arXiv:0910.5496] [INSPIRE].

[28] R. Donagi, A. Grassi and E. Witten, A Nonperturbative superpotential with $E_{8}$ symmetry, Mod. Phys. Lett. A 11 (1996) 2199 [hep-th/9607091] [InSPIRE].

[29] G. Curio and D. Lüst, A Class of $N=1$ dual string pairs and its modular superpotential, Int. J. Mod. Phys. A 12 (1997) 5847 [hep-th/9703007] [INSPIRE]. 
[30] A.P. Braun, M. Del Zotto, J. Halverson, M. Larfors, D.R. Morrison and S. Schäfer-Nameki, Infinitely many M2-instanton corrections to M-theory on $G_{2}$-manifolds, JHEP 09 (2018) 077 [arXiv: 1803.02343] [INSPIRE].

[31] J. Distler, Resurrecting (2,0) compactifications, Phys. Lett. B 188 (1987) 431 [InSPIRE].

[32] E. Silverstein and E. Witten, Criteria for conformal invariance of $(0,2)$ models, Nucl. Phys. B 444 (1995) 161 [hep-th/9503212] [INSPIRE].

[33] C. Beasley and E. Witten, Residues and world sheet instantons, JHEP 10 (2003) 065 [hep-th/0304115] [INSPIRE].

[34] E.I. Buchbinder and B.A. Ovrut, Non-vanishing Superpotentials in Heterotic String Theory and Discrete Torsion, JHEP 01 (2017) 038 [arXiv:1611.01922] [INSPIRE].

[35] E. Buchbinder, A. Lukas, B. Ovrut and F. Ruehle, Heterotic Instanton Superpotentials from Complete Intersection Calabi-Yau Manifolds, JHEP 10 (2017) 032 [arXiv:1707.07214] [INSPIRE].

[36] E.I. Buchbinder, A. Lukas, B.A. Ovrut and F. Ruehle, Heterotic Instantons for Monad and Extension Bundles, JHEP 02 (2020) 081 [arXiv:1912.07222] [INSPIRE].

[37] E.I. Buchbinder, A. Lukas, B.A. Ovrut and F. Ruehle, Instantons and Hilbert Functions, arXiv: 1912.08358 [INSPIRE].

[38] L. Kamenova and C. Vafa, Kobayashi non-hyperbolicity of Calabi-Yau manifolds via mirror symmetry, arXiv: 1908.08573.

[39] M. Billó, M. Frau, I. Pesando, F. Fucito, A. Lerda and A. Liccardo, Classical gauge instantons from open strings, JHEP 02 (2003) 045 [hep-th/0211250] [INSPIRE].

[40] N. Akerblom, R. Blumenhagen, D. Lüst and M. Schmidt-Sommerfeld, Instantons and Holomorphic Couplings in Intersecting D-brane Models, JHEP 08 (2007) 044 [arXiv:0705.2366] [INSPIRE].

[41] M. Aganagic, C. Beem and S. Kachru, Geometric transitions and dynamical SUSY breaking, Nucl. Phys. B 796 (2008) 1 [arXiv:0709.4277] [INSPIRE].

[42] C. Petersson, Superpotentials From Stringy Instantons Without Orientifolds, JHEP 05 (2008) 078 [arXiv: 0711.1837] [INSPIRE].

[43] R. Argurio, M. Bertolini, S. Franco and S. Kachru, Meta-stable vacua and D-branes at the conifold, JHEP 06 (2007) 017 [hep-th/0703236] [INSPIRE].

[44] R. Argurio, M. Bertolini, G. Ferretti, A. Lerda and C. Petersson, Stringy instantons at orbifold singularities, JHEP 06 (2007) 067 [arXiv:0704.0262] [INSPIRE].

[45] M. Bianchi, F. Fucito and J.F. Morales, D-brane instantons on the $T^{6} / Z(3)$ orientifold, JHEP 07 (2007) 038 [arXiv:0704.0784] [INSPIRE].

[46] L.E. Ibáñez, A.N. Schellekens and A.M. Uranga, Instanton Induced Neutrino Majorana Masses in CFT Orientifolds with MSSM-like spectra, JHEP 06 (2007) 011 [arXiv:0704.1079] [INSPIRE].

[47] R. Blumenhagen, M. Cvetič, R. Richter and T. Weigand, Lifting D-Instanton Zero Modes by Recombination and Background Fluxes, JHEP 10 (2007) 098 [arXiv:0708.0403] [INSPIRE].

[48] I. Garcia-Etxebarria and A.M. Uranga, Non-perturbative superpotentials across lines of marginal stability, JHEP 01 (2008) 033 [arXiv:0711.1430] [INSPIRE]. 
[49] I. Garcia-Etxebarria, F. Marchesano and A.M. Uranga, Non-perturbative F-terms across lines of BPS stability, JHEP 07 (2008) 028 [arXiv: 0805.0713] [INSPIRE].

[50] M. Cvetič, I. Garcia-Etxebarria and R. Richter, Branes and instantons at angles and the F-theory lift of O(1) instantons, AIP Conf. Proc. 1200 (2010) 246 [arXiv:0911.0012] [INSPIRE].

[51] M. Cvetič, I. Garcia Etxebarria and J. Halverson, Three Looks at Instantons in F-theory New Insights from Anomaly Inflow, String Junctions and Heterotic Duality, JHEP 11 (2011) 101 [arXiv: 1107.2388] [INSPIRE].

[52] V. Braun, On Free Quotients of Complete Intersection Calabi-Yau Manifolds, JHEP 04 (2011) 005 [arXiv: 1003.3235] [INSPIRE].

[53] C. Beasley and E. Witten, New instanton effects in supersymmetric QCD, JHEP 01 (2005) 056 [hep-th/0409149] [INSPIRE].

[54] C. Beasley and E. Witten, New instanton effects in string theory, JHEP 02 (2006) 060 [hep-th/0512039] [INSPIRE].

[55] P.G. Camara, L.E. Ibáñez and F. Marchesano, RR photons, JHEP 09 (2011) 110 [arXiv:1106.0060] [INSPIRE].

[56] C. Mayrhofer, E. Palti, O. Till and T. Weigand, On Discrete Symmetries and Torsion Homology in F-theory, JHEP 06 (2015) 029 [arXiv:1410.7814] [INSPIRE].

[57] R. Donagi and K. Wendland, On orbifolds and free fermion constructions, J. Geom. Phys. 59 (2009) 942 [arXiv: 0809.0330] [INSPIRE].

[58] C. Vafa and E. Witten, On orbifolds with discrete torsion, J. Geom. Phys. 15 (1995) 189 [hep-th/9409188] [INSPIRE].

[59] C. Angelantonj, I. Antoniadis, G. D'Appollonio, E. Dudas and A. Sagnotti, Type I vacua with brane supersymmetry breaking, Nucl. Phys. B $\mathbf{5 7 2}$ (2000) 36 [hep-th/9911081] [INSPIRE].

[60] R. Blumenhagen, D. Lüst and T.R. Taylor, Moduli stabilization in chiral type IIB orientifold models with fluxes, Nucl. Phys. B 663 (2003) 319 [hep-th/0303016] [INSPIRE].

[61] J.P. Conlon and L.T. Witkowski, Scattering and Sequestering of Blow-Up Moduli in Local String Models, JHEP 12 (2011) 028 [arXiv:1109.4153] [INSPIRE].

[62] D. Friedan, E.J. Martinec and S.H. Shenker, Conformal Invariance, Supersymmetry and String Theory, Nucl. Phys. B 271 (1986) 93 [inSPIRE].

[63] J.P. Conlon, M. Goodsell and E. Palti, Anomaly Mediation in Superstring Theory, Fortsch. Phys. 59 (2011) 5 [arXiv: 1008.4361] [INSPIRE].

[64] F. Denef, M.R. Douglas, B. Florea, A. Grassi and S. Kachru, Fixing all moduli in a simple F-theory compactification, Adv. Theor. Math. Phys. 9 (2005) 861 [hep-th/0503124] [INSPIRE].

[65] D. Lüst, S. Reffert, W. Schulgin and S. Stieberger, Moduli stabilization in type IIB orientifolds (I): Orbifold limits, Nucl. Phys. B 766 (2007) 68 [hep-th/0506090] [InSPIRE].

[66] R. Blumenhagen, M. Cvetič, F. Marchesano and G. Shiu, Chiral D-brane models with frozen open string moduli, JHEP 03 (2005) 050 [hep-th/0502095] [INSPIRE].

[67] D. Lüst, S. Reffert, E. Scheidegger, W. Schulgin and S. Stieberger, Moduli Stabilization in Type IIB Orientifolds (II), Nucl. Phys. B 766 (2007) 178 [hep-th/0609013] [INSPIRE]. 
[68] M. Bershadsky, S. Cecotti, H. Ooguri and C. Vafa, Kodaira-Spencer theory of gravity and exact results for quantum string amplitudes, Commun. Math. Phys. 165 (1994) 311 [hep-th/9309140] [INSPIRE].

[69] S. Ferrara, J.A. Harvey, A. Strominger and C. Vafa, Second quantized mirror symmetry, Phys. Lett. B 361 (1995) 59 [hep-th/9505162] [INSPIRE].

[70] A. Klemm and M. Mariño, Counting BPS states on the enriques Calabi-Yau, Commun. Math. Phys. 280 (2008) 27 [hep-th/0512227] [INSPIRE].

[71] A.-K. Kashani-Poor, R. Minasian and H. Triendl, Enhanced supersymmetry from vanishing Euler number, JHEP 04 (2013) 058 [arXiv: 1301.5031] [INSPIRE].

[72] B. Haghighat, S. Murthy, C. Vafa and S. Vandoren, F-Theory, Spinning Black Holes and Multi-string Branches, JHEP 01 (2016) 009 [arXiv: 1509.00455] [INSPIRE].

[73] C. Lawrie, S. Schäfer-Nameki and T. Weigand, Chiral $2 d$ theories from N $=4 S Y M$ with varying coupling, JHEP 04 (2017) 111 [arXiv: 1612.05640] [INSPIRE].

[74] P.H. Ginsparg, Applied conformal field theory, in Les Houches Summer School in Theoretical Physics: Fields, Strings, Critical Phenomena, Les Houches France (1988), pg. 1 [hep-th/9108028] [INSPIRE].

[75] S. Hamidi and C. Vafa, Interactions on Orbifolds, Nucl. Phys. B 279 (1987) 465 [inSPIRE].

[76] N. Arkani-Hamed, L. Motl, A. Nicolis and C. Vafa, The String landscape, black holes and gravity as the weakest force, JHEP 06 (2007) 060 [hep-th/0601001] [INSPIRE].

[77] B. Heidenreich, M. Reece and T. Rudelius, Sharpening the Weak Gravity Conjecture with Dimensional Reduction, JHEP 02 (2016) 140 [arXiv: 1509.06374] [INSPIRE].

[78] E. Palti, The Weak Gravity Conjecture and Scalar Fields, JHEP 08 (2017) 034 [arXiv: 1705. 04328] [INSPIRE].

[79] S.-J. Lee, W. Lerche and T. Weigand, A Stringy Test of the Scalar Weak Gravity Conjecture, Nucl. Phys. B 938 (2019) 321 [arXiv:1810.05169] [INSPIRE].

[80] H. Ooguri and C. Vafa, Non-supersymmetric AdS and the Swampland, Adv. Theor. Math. Phys. 21 (2017) 1787 [arXiv: 1610.01533] [INSPIRE].

[81] S. Cecotti, Special Geometry and the Swampland, to appear.

[82] A. Sen, Orientifold limit of F-theory vacua, Phys. Rev. D 55 (1997) 7345 [hep-th/9702165] [INSPIRE].

[83] D.R. Morrison and W. Taylor, Sections, multisections and U(1) fields in F-theory, arXiv: 1404.1527 [INSPIRE].

[84] C. Mayrhofer, E. Palti, O. Till and T. Weigand, Discrete Gauge Symmetries by Higgsing in four-dimensional F-theory Compactifications, JHEP 12 (2014) 068 [arXiv:1408.6831] [INSPIRE].

[85] R. Friedman, J. Morgan and E. Witten, Vector bundles and F-theory, Commun. Math. Phys. 187 (1997) 679 [hep-th/9701162] [INSPIRE].

[86] L.B. Anderson and W. Taylor, Geometric constraints in dual F-theory and heterotic string compactifications, JHEP 08 (2014) 025 [arXiv: 1405.2074] [INSPIRE].

[87] L.B. Anderson, F. Apruzzi, X. Gao, J. Gray and S.-J. Lee, Instanton superpotentials, Calabi-Yau geometry and fibrations, Phys. Rev. D 93 (2016) 086001 [arXiv:1511.05188] [INSPIRE]. 
[88] P.G. Camara, E. Dudas, T. Maillard and G. Pradisi, String instantons, fluxes and moduli stabilization, Nucl. Phys. B $\mathbf{7 9 5}$ (2008) 453 [arXiv:0710.3080] [InSPIRE].

[89] P.G. Camara and E. Dudas, Multi-instanton and string loop corrections in toroidal orbifold models, JHEP 08 (2008) 069 [arXiv: 0806.3102] [inSPIRE].

[90] P.G. Camara, C. Condeescu, E. Dudas and M. Lennek, Non-perturbative Vacuum Destabilization and D-brane Dynamics, JHEP 06 (2010) 062 [arXiv:1003.5805] [INSPIRE].

[91] M. Bertolini and M.R. Plesser, Worldsheet instantons and $(0,2)$ linear models, JHEP 08 (2015) 081 [arXiv: 1410.4541] [INSPIRE].

[92] E. Witten, More On Superstring Perturbation Theory: An Overview Of Superstring Perturbation Theory Via Super Riemann Surfaces, arXiv:1304.2832 [INSPIRE].

[93] J.J. Atick, L.J. Dixon and A. Sen, String Calculation of Fayet-Iliopoulos d Terms in Arbitrary Supersymmetric Compactifications, Nucl. Phys. B 292 (1987) 109 [INSPIRE].

[94] G. Aldazabal and L.E. Ibáñez, A Note on $4 D$ Heterotic String Vacua, FI-terms and the Swampland, Phys. Lett. B 782 (2018) 375 [arXiv:1804.07322] [InSPIRE].

[95] R. Pius, A. Rudra and A. Sen, String Perturbation Theory Around Dynamically Shifted Vacuum, JHEP 10 (2014) 070 [arXiv:1404.6254] [INSPIRE].

[96] A. Sen, Supersymmetry Restoration in Superstring Perturbation Theory, JHEP 12 (2015) 075 [arXiv: 1508.02481] [INSPIRE].

[97] L.B. Anderson, X. Gao, J. Gray and S.-J. Lee, Fibrations in CICY Threefolds, JHEP 10 (2017) 077 [arXiv: 1708.07907] [inSPIRE].

[98] L.B. Anderson, J. Gray, A. Lukas and E. Palti, Two Hundred Heterotic Standard Models on Smooth Calabi-Yau Threefolds, Phys. Rev. D 84 (2011) 106005 [arXiv:1106.4804] [INSPIRE].

[99] L.B. Anderson, J. Gray, A. Lukas and E. Palti, Heterotic Line Bundle Standard Models, JHEP 06 (2012) 113 [arXiv:1202.1757] [InSPIRE].

[100] L.B. Anderson, A. Constantin, J. Gray, A. Lukas and E. Palti, A Comprehensive Scan for Heterotic SU(5) GUT models, JHEP 01 (2014) 047 [arXiv:1307.4787] [INSPIRE].

[101] S. Groot Nibbelink, O. Loukas, F. Ruehle and P.K.S. Vaudrevange, Infinite number of MSSMs from heterotic line bundles?, Phys. Rev. D 92 (2015) 046002 [arXiv:1506.00879] [INSPIRE].

[102] R. Blumenhagen, G. Honecker and T. Weigand, Loop-corrected compactifications of the heterotic string with line bundles, JHEP 06 (2005) 020 [hep-th/0504232] [INSPIRE].

[103] L.B. Anderson, Y.-H. He and A. Lukas, Heterotic Compactification, An Algorithmic Approach, JHEP 07 (2007) 049 [hep-th/0702210] [INSPIRE].

[104] G. Papadopoulos and P.K. Townsend, Compactification of $D=11$ supergravity on spaces of exceptional holonomy, Phys. Lett. B 357 (1995) 300 [hep-th/9506150] [INSPIRE].

[105] J.A. Harvey and G.W. Moore, Superpotentials and membrane instantons, hep-th/9907026 [INSPIRE].

[106] B.S. Acharya, M theory, Joyce orbifolds and superYang-Mills, Adv. Theor. Math. Phys. 3 (1999) 227 [hep-th/9812205] [INSPIRE].

[107] A. Kovalev, Twisted connected sums and special Riemannian holonomy, J. Reine Angew. Math. 565 (2003) 125 [math/0012189]. 
[108] A. Kovalev and N.-H. Lee, K3 surfaces with non-symplectic involution and compact irreducible $G_{2}$-manifolds, Math. Proc. Camb. Philos. Soc. 151 (2011) 193.

[109] A. Corti, M. Haskins, J. Nordstrom and T. Pacini, Asymptotically cylindrical Calabi-Yau 3-folds from weak fano 3-folds, Geom. Topol. 17 (2013) 1955 [arXiv:1206.2277].

[110] D.D. Joyce, Compact Riemannian 7-manifolds with holonomy $G_{2}$. II, J. Diff. Geom. 43 (1996) 329.

[111] A. Corti, M. Haskins, J. Nordstrom and T. Pacini, $\mathrm{G}_{2}$-manifolds and associative submanifolds via semi-fano 3-folds, Duke Math. J. 164 (2015) 1971.

[112] G. Menet, J. Nordstrom and H. Sá Earp, Construction of $G_{2}$-instantons via twisted connected sums, arXiv:1510.03836 [INSPIRE].

[113] H. Sá Earp and T. Walpuski, $G_{2}$-instantons over twisted connected sums, Geom. Topol. 19 (2015) 1263.

[114] H. Sá Earp, $G_{2}$-instantons over asymptotically cylindrical manifolds, Geom. Topol. 19 (2015) 61.

[115] T. Walpuski, $G_{2}$-instantons on generalised kummer constructions, Geom. Topol. 17 (2013) 2345.

[116] T. Walpuski, $\mathrm{G}_{2}$-instantons over twisted connected sums: An example, Math. Res. Lett. 23 (2016) 529 [arXiv: 1505.01080].

[117] T. Walpuski, $G_{2}$-instantons, associative submanifolds and fueter sections, Commun. Anal. Geom. 25 (2017) 847 [arXiv: 1205.5350].

[118] D. Joyce, Conjectures on counting associative 3-folds in g2-manifolds, in Modern Geometry: A Celebration of the Work of Simon Donaldson, AMS Press, Providence U.S.A. (2018), pg. 97 [arXiv: 1610.09836].

[119] J. Halverson and D.R. Morrison, On gauge enhancement and singular limits in $G_{2}$ compactifications of M-theory, JHEP 04 (2016) 100 [arXiv:1507.05965] [INSPIRE].

[120] A.P. Braun and S. Schäfer-Nameki, Compact, Singular $G_{2}$-Holonomy Manifolds and M/Heterotic/F-Theory Duality, JHEP 04 (2018) 126 [arXiv:1708.07215] [INSPIRE].

[121] B.S. Acharya, A.P. Braun, E.E. Svanes and R. Valandro, Counting associatives in compact $G_{2}$ orbifolds, JHEP 03 (2019) 138 [arXiv: 1812.04008] [INSPIRE].

[122] A.P. Braun, M-Theory and Orientifolds, arXiv:1912.06072 [INSPIRE].

[123] O. DeWolfe, A. Giryavets, S. Kachru and W. Taylor, Enumerating flux vacua with enhanced symmetries, JHEP 02 (2005) 037 [hep-th/0411061] [INSPIRE].

[124] M. Dine and Z. Sun, R symmetries in the landscape, JHEP 01 (2006) 129 [hep-th/0506246] [INSPIRE].

[125] E. Palti, Low Energy Supersymmetry from Non-Geometry, JHEP 10 (2007) 011 [arXiv:0707.1595] [INSPIRE].

[126] A.-K. Kashani-Poor and A. Tomasiello, A stringy test of flux-induced isometry gauging, Nucl. Phys. B 728 (2005) 135 [hep-th/0505208] [INSPIRE]. 